\title{
Whole-genome characterization of Rosa chinensis AP2/ERF transcription factors and analysis of negative regulator RCDREB2B in Arabidopsis
}

Wei $\mathrm{Li}^{\dagger}$, Ziwen Geng ${ }^{\dagger}$, Cuiping Zhang, Kuiling Wang and Xinqiang Jiang*

\begin{abstract}
Background: Rose (Rosa chinensis) is a traditional famous flower with valuable ornamental characteristics. However, drought stress restricts its growth and development, leading to an abnormal phenotype. One of the main transcription factor (TF) protein groups in the plant kingdom are the APETALA2/ethylene-responsive factor (AP2/ ERF) proteins and are potentially involved in the growth and stress responses of various plants.

Results: Our investigation mainly focused on exploring the genome of rose and thereby we discovered 135 apparent AP2/ERF TFs. Phylogenic analyses revealed that RCAP2/ERF genes are categorized into DREB, Soloist, AP2, and ERF subfamilies, and are further classified these into 17 groups, with the same as Malus domestica and Arabidopsis thaliana. The analysis of the gene structure revealed that the introns ranged from 0 to 9 in number. Pattern examination demonstrated that the RCAP2/ERF predominantly consists of typical AP2 domains, of which the 2nd motif is the most ubiquitous. Distributions of cis-acting elements indicated that members of the AP2/ERF family are frequently involved in growth and development, phytohormone and stress response in rose species. Also, the distribution mapping of the rose chromosomes indicated that AP2/ERF class genes are dispersed among all seven chromosomes. Additionally, we isolated a novel DREB A2 subgroup gene and named it RCDREB2B. Subsequently, the RCDREB2B transcript accumulation was repressed under the mild and severe drought stress in the root samples of rose. RcDREB2B was targeted to the nucleus and exhibited transactivation in yeast cells. The overexpression of $R C D R E B 2 B$ was found to promote sensitivity to a higher salt concentration, $A B A$, and PEG at the germination and post-germination stages. Twelve putative osmotic and ABA-related genes were impaired in RCDREB2Boverexpressing plants.
\end{abstract}

Conclusions: The results provide comprehensive information regarding the gene structure, phylogenic, and distribution of the rose AP2/ERF family and bring insight into the complex transcriptional gene regulation of RCAP2/ $E R F$. Findings in this study would also contribute to further understanding of the RCDREB2B gene in rose.

Keywords: Rose, AP2/ERF, Transcription factor, RCDREB2B, Drought

\footnotetext{
* Correspondence: jiangxinqiang8@163.com

${ }^{\dagger}$ Wei Li and Ziwen Geng contributed equally to this work.

College of Landscape Architecture and Forestry, Qingdao Agricultural

University, Qingdao 266000, China
}

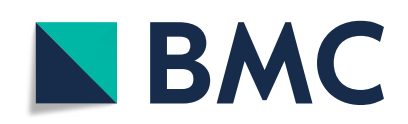

(c) The Author(s). 2021 Open Access This article is licensed under a Creative Commons Attribution 4.0 International License, which permits use, sharing, adaptation, distribution and reproduction in any medium or format, as long as you give appropriate credit to the original author(s) and the source, provide a link to the Creative Commons licence, and indicate if changes were made. The images or other third party material in this article are included in the article's Creative Commons licence, unless indicated otherwise in a credit line to the material. If material is not included in the article's Creative Commons licence and your intended use is not permitted by statutory regulation or exceeds the permitted use, you will need to obtain permission directly from the copyright holder. To view a copy of this licence, visit http://creativecommons.org/licenses/by/4.0/. The Creative Commons Public Domain Dedication waiver (http://creativecommons.org/publicdomain/zero/1.0/) applies to the data made available in this article, unless otherwise stated in a credit line to the data. 


\section{Background}

Transcription factors (TFs) mediate plant developmental and growth processes. Besides, they also perform vital roles in transmitting stimulatory or inhibitory signals $[1$, 2]. APETALA2/ethylene-responsive factor (AP2/ERF) is one of the major TF superfamilies in plants. They possess either single or double AP2 DNA-binding domains that have a sequence homology of approximately 70 amino acid residues [3]. According to AP2 domain numbers and sequence similarity, AP2/ERF is classified into three subgroups, namely ERF, RAV, and AP2 gene subfamilies [3]. Whereas, the ERF subfamily contains a single AP2 domain while the AP2 subfamily has two. Both an AP2 domain and a B3 DNA-binding domain are present in the RAV subgroup [4]. Also, based on the variations in binding promoter sequences of the ERF family can be split into DREB and ERF subfamilies [5]. Arabidopsis thaliana has DREB and the ERF subfamily was additionally split into six segments.

AP2/ERF TFs have been shown to regulate a variety of processes in plants [6]. For example, they regulate plant's responses to abiotic stress including salt, drought, and cold in addition to normal growth and development [79]. AP2 domains were initially described as recurring motifs inside AP2 proteins of $A$. thaliana that mediated a regulatory function during development [3]. Four DNA-binding proteins stimulated by ethylene in tobacco have been implicated in gene transcription, which demonstrated that ERF domains were conserved [9]. ERF proteins were later established to have other roles in several different plants [10]. A total of 145 AP2/ERF are now well recognized in Arabidopsis including 64 from the ERF subfamily, 56 from the DREB subfamily, six from RAV, one from the Soloist, and 17 from the AP2 subfamily [5].

Rose (Rosa chinensis) is a well-known traditional wooden plant worldwide. Additionally, it is also one of the most important horticultural crops with high economic and ornamental value and has been used for the breeding of cut roses across the globe. With the genome sequencing of diploid Rosa chinensis 'Old Blush', it can presumably become an excellent model plant for functional genomics of ornamental plants [11]. Many gene families with corresponding growth and development need to be examined. In plants, AP2/ERF proteins are profoundly involved in controlling a plant's growth and development. With more extensive genomic DNA sequencing being carried out for numerous plants, large number of AP2/ERF proteins have been recognized as yet, including Apium graveolens [12], pear (Pyrus communis) [13], cauliflower (Brassica oleracea) [14], pepper (Capsicum annuum) [15], Salvia miltiorrhiza [16], Musa species [17], Chinese jujube (Ziziphus jujuba) [18], buckwheat (Fagopyum tataricum) [19] and peach (Prunus persica) [20]. However, so far, none of the research studies have been previously conducted on the detection and characterization of AP2/ERF TFs in rose. Therefore, due to the critical contributions of AP2/ERF genes in different biological reactions, a comprehensive study of AP2/ ERF in rose is extremely essential.

Initially, our investigation first identified and examined gene sequences, motif structure, promoters, and location of rose AP2/ERF genes on each chromosome. Then, gene duplication and evolutionary mechanisms were subsequently analyzed. Importantly, we also identified a novel DREB A2 subgroup member (i.e., RcDREB2B) in rose. RcDREB2B is a nuclear protein and has transactivation in yeast cells. RcDREB2B was repressed under mild and severe drought stress in rose root samples. The overexpression of $R c D R E B 2 B$ in Arabidopsis exhibited enhanced sensitivity to high salt, ABA, and PEG at germination and post-germination stages. Osmotic and ABA-related genes expressions were impaired in $R c D R E B 2 B$ transgenic plants. Our findings demonstrated the regulatory roles of rose AP2/ERF TFs in normal homeostasis, which will not only provide a fresh insight into the evolutionary mechanism of this particular TF family in plants but will also contribute towards revealing the molecular mechanisms of development and stress response in rose and other species.

\section{Results}

\section{Identification and analysis of RCAP2/ERF}

We used BLASTP and HMMER3.1 in the entire rose genome to discover AP2/ERF members. All potential rose proteins were then subjected to domain analysis to confirm the presence of the AP2 protein domain (PF00847). Eventually, a total of 135 AP2/ERF family members were identified in rose. According to the distribution order of their chromosomal locations, they were named consecutively from RcAP2/ERF1 to RcAP2/ ERF135. Their characteristics including gene name, locus ID, physical position, and other properties are shown in Table S1. RcAP2/ERF segregated to 7 rose chromosomes and mainly localized in the nucleus, chloroplast, cytoplasm, and mitochondria. The longest sequence with 832 amino acid residues was RcAP2/ERF52, whereas the shortest having 125 amino acids was RcAP2/ERF132. The predicted isoelectric points ranged from 4.52 (RcAP2/ERF54) to 10.28 (RcAP2/ERF99). The protein molecular weight varied between $13.94 \mathrm{kDa}$ to 91.01 $\mathrm{kDa}$. Our findings revealed that different biological roles were played by individual coding regions of $R c A P 2 / E R F s$.

\section{RCAP2/ERF gene structure}

The gene structure of $R c A P 2 / E R F$ is closely related to its function, and together with their phylogenetic analysis, could reflect the phylogenetic relationships among the 
$R c A P 2 / E R F$. As shown in Fig. 1, the RcAP2/ERF family was divided into seven groups. Analysis of RcAP2/ERF structure for exon/intron organizations revealed that each gene had 0 to 9 introns (Fig. 1), the genes clustered into the same branch on the phylogenetic tree were found with similar exon-intron structure. The results provided support validating our analysis regarding gene structure. Further, most RcAP2/ERF (94, 69.62\%) contained no introns, only 19 (14.07\%) contained one intron whereas RcAP2/ERF30 contained nine introns. It was surprising yet interesting that none of the $R c A P 2 / E R F$ contained three introns. AP2 gene configuration was reasonably conserved among family members, and 51\% of AP2/ERF genes had no intron (Fig. 1). Typically, closest family members had a common exon/intron configuration concerning intron number and phase as well as the length of exons.

In an attempt to carry out the in-depth examination of typical RcAP2/ERF protein sequences, we assessed 135 typical arrangements from the MEME website. We predicted that the conserved sequences would amount to 10 in number their sequences were sought (Fig. 1). Besides, we also observed that common patterns with the same orientation and location were most apparent among phylogenetically close relatives. This suggests common roles for AP2 members located in similar subgroups. Motifs 1 and 7, with a net presence of $61.48 \%$ $(83 / 135)$ and $60.74 \%(82 / 135)$ respectively were frequent among RcAP2/ERF. Several preserved motifs have been identified in various subgroups. Phylogenetic investigations show similarity in gene composition and pattern arrangements of subgroup members adding confidence to classification accuracy.

\section{Phylogenetic and evolutionary analysis of RCAP2/ERF}

To assess phylogenetic associations of rose AP2/ERF, we aligned AP2 domain sequences of rose, apple (Malus demostica), and Arabidopsis to construct a parallel phylogenetic tree [21]. One hundred thirty-five RcAP2/ ERF proteins of rose, 260 members from apple, and 167

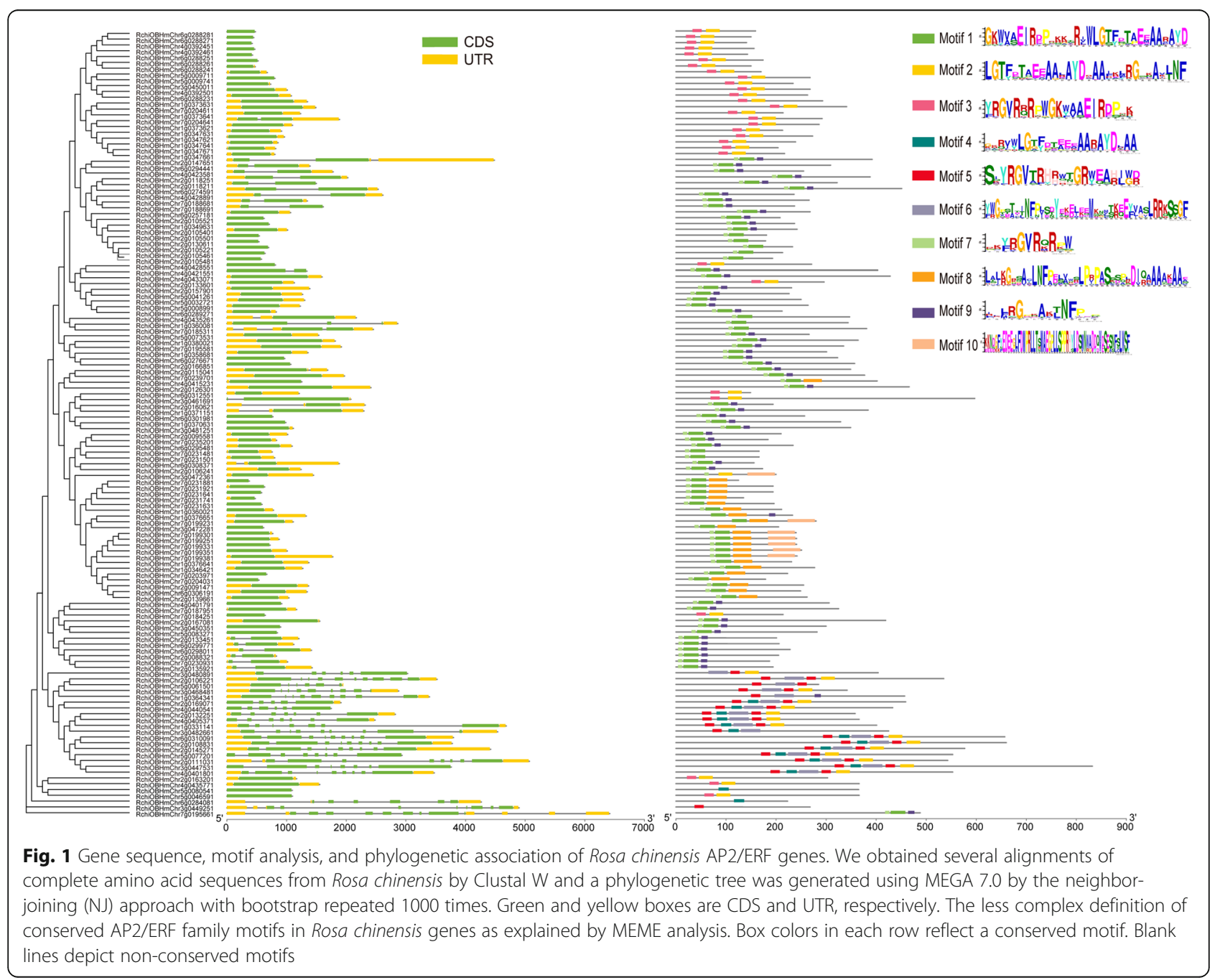


members from Arabidopsis were selected for phylogenic analysis (Fig. 2). Using the same criteria for classification as in Arabidopsis, the AP2/ERF proteins of rose were classified into DREB, Solosist, AP2, and ERF segments containing 44, 6, 17, and 68 RcAP2/ERF proteins, respectively (Table 1). The RcAP2/ERF gene members within groups were mostly the same except ERF-B1, ERF-B6, and ERF-B3. DREB segment is further split into six subgroups, from A1 to A6, containing 7, 11, 1, 15, 7,
$3 \mathrm{AP} 2 / \mathrm{ERF}$ proteins in rose and 3, 25, 2, 18, 18, $10 \mathrm{AP} 2 /$ ERF members in apple, respectively. ERF subfamily being the most abundant type in rose, accounted for $50.37 \%$ of all RcAP2/ERF proteins, which was closely similar to apple, with a percentage of $51.54 \%$. Arabidopsis has the lowest percentage of ERF subfamily members, which accounted for $46.11 \%$. ERF subfamily was also further segregated to B1-6 subgroups, containing $12,3,13,15$, 5 , and 20 RcAP2/ERF proteins, respectively. AP2

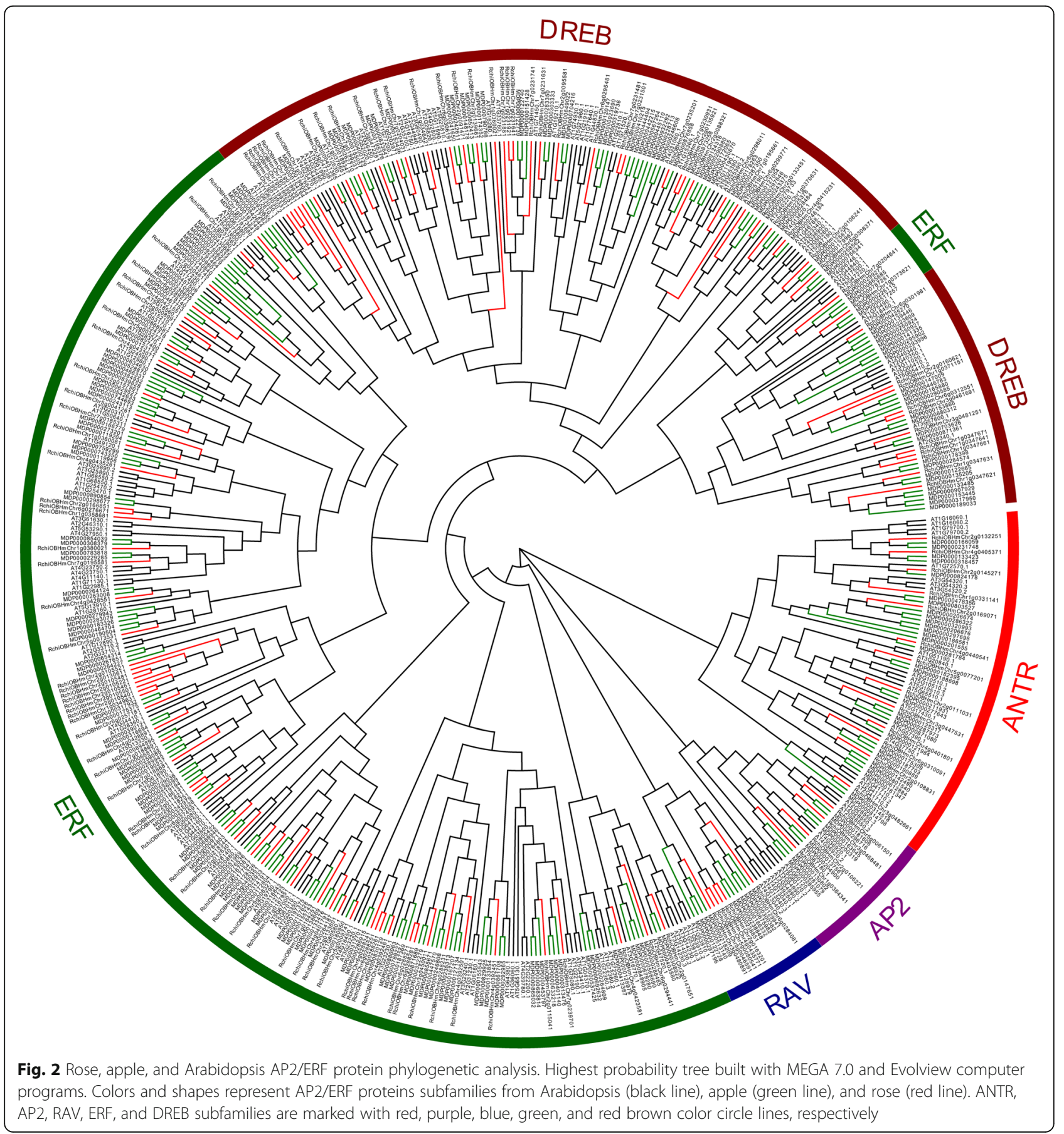


Table 1 Summary of AP2/ERF transcription factors of Rosa chinensis, Malus domestica and Arabidopsis thaliana

\begin{tabular}{|c|c|c|c|c|}
\hline \multirow[t]{2}{*}{ Classification } & \multirow[t]{2}{*}{ Group } & \multicolumn{3}{|l|}{ Number } \\
\hline & & Rosa chinensis & Malus domestica & Arabidospis thaliana \\
\hline \multirow[t]{6}{*}{ DREB subfamily } & $\mathrm{A} 1$ & 7 & 3 & 7 \\
\hline & $\mathrm{A} 2$ & 11 & 25 & 9 \\
\hline & A3 & 1 & 2 & 1 \\
\hline & A4 & 15 & 18 & 16 \\
\hline & A5 & 7 & 18 & 15 \\
\hline & A6 & 3 & 10 & 10 \\
\hline \multirow[t]{6}{*}{ EFR subfamily } & B1 & 12 & 38 & 18 \\
\hline & B2 & 3 & 11 & 11 \\
\hline & B3 & 13 & 25 & 11 \\
\hline & B4 & 15 & 18 & 12 \\
\hline & B5 & 5 & 6 & 8 \\
\hline & B6 & 20 & 36 & 17 \\
\hline \multirow[t]{3}{*}{ AP2 subfamily } & AP2-R1 & 4 & 9 & 11 \\
\hline & ANTR1 & 6 & 15 & 8 \\
\hline & ANTR2 & 7 & 16 & 10 \\
\hline Solosist subfamily & & 6 & 10 & 3 \\
\hline Total AP2/ERF family factors & & 135 & 260 & 167 \\
\hline
\end{tabular}

subfamily contains 17 members of RcAP2/ERF proteins, whereas the Solosist subfamily is the smallest, consisting of only 6 members, indicating that $R c A P 2 / E R F$ genes were distributed in different clades unevenly.

\section{Chromosomal location and gene duplication of RcAP2/ ERFs}

Mapchart software was used to identify the $R c A P 2 / E R F$ chromosomal location. All RcAP2/ERFs had precise positions on the chromosomes. Each rose chromosome contains more than 11 RcAP2/ERFs (Fig. 3a). The $R c A P 2 / E R F$ genes on 7 chromosomes are randomly and unevenly distributed. Our maximum predicted the number of $R c A P 2 / E R F$ was 38 genes on chromosome 2 and can be compared to rather fewer, 11 genes located on chromosomes 5 and 3 . Although chromosome 3 is the shortest and chromosome 5 is the longest in rose they contain the least $R c A P 2 / E R F$. Therefore, there is no apparent correlation between chromosome length and RcAP2/ERF gene distribution.

Segmental and tandem duplication are evolutionary mechanisms that result in generating groups of genes [22]. Hence, Synteny and MCScanX [23] were used to analyze the duplication and segmental events of RcAP2/ $E R F$. As shown in Fig. 3b, 20 genes were confirmed as tandem duplicated genes. Chromosomes 7 and 4 had three groups of two duplicate tandem genes, while chromosomes 6 and 1 had five and four duplicate tandem genes respectively. Segmental duplication events were also detected in each chromosome and accounted for
$24.4 \%$ of the RcAP2/ERFs. Taken in combination, these outcomes indicated that segmental and tandem duplication extensively contributes to the expansion of the RcAP2/ERF family, whereas the former is being more deeply involved in particular.

\section{Analyzing cis-regulatory motifs in promoters of RcAP2/ERF genes}

To establish the gene expression control of $R c A P 2 / E R F$, a bioinformatics review was performed to detect apparent cis-regulatory regions in the $R c A P 2 / E R F$ gene promoter region. This promoter zone was identified by the $\sim 1500 \mathrm{bp}$ upstream area of the initial transcript sites. Figure 4 illustrates that different promoter cis-elements were grouped into three groups, including plant growth and development (MBSI, E2FB, MSA-like, P-box, TCAelement, AT-rich element,), phytohormone responsive (ABRE, CGTCA-motif, ERE, TGACG-motif, AuxRR, GARE-motif, TATC-box), and abiotic and biotic stresses (MBS, TC-rich repeats, LTR, DRE, WUN-motif, MYC, W-box, MYB, STRE, WRE3). These cis-regulatory elements were found to be randomly dispersed among various promoters of $R c A P 2 / E R F$ genes. ERF subfamily B1 group member RchiOBHmChr5g0008991 has the largest number of cis-elements, accounting for 43. MYB and MYC elements were present in $79.4 \%$ of $R c A P 2 / E R F$ gene promoters, whereas $77.9 \%$ were found with ABRE. Moreover, 66.2, 50.7, 45.6, and 44.9\% of RcAP2/ERF gene promoters were presenting MeJA, gibberellin, W box and MBS, respectively, which indicating important 


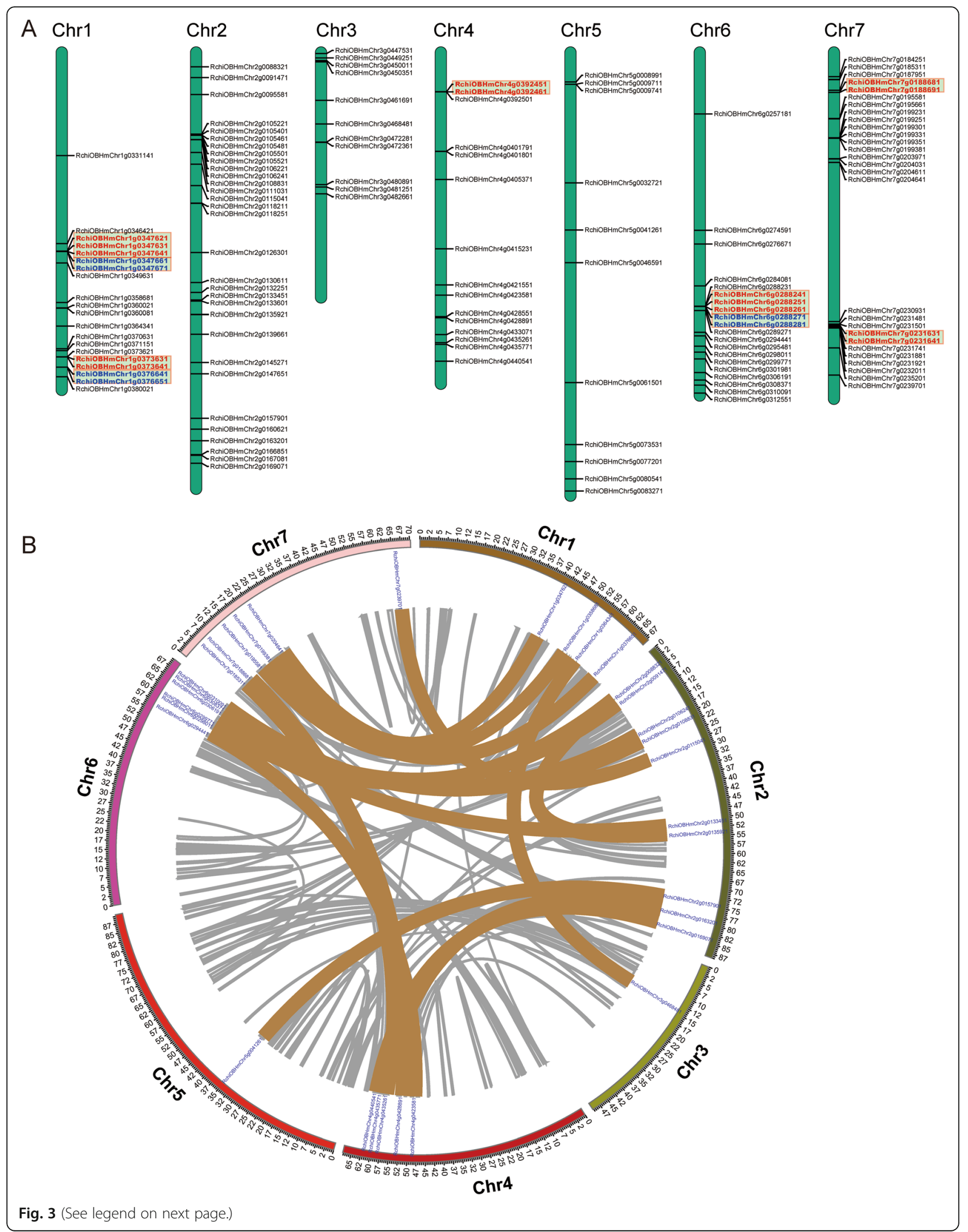


(See figure on previous page.)

Fig. 3 Chromosome mapping and collinearity analysis of RCAP2/ERF genes. a RcAP2/ERF genes distributed on seven rose chromosomes. The brown colored line between the two gene names indicated that they were tandem repeat gene pairs. Gene locations are shown by the scale. The Gene location on each chromosome is represented by a line. b Collinearity analysis AP2/ERF gene family in the rose genome. All rose AP2/ ERF family duplicated gene pairs are depicted in the rose chromosomes (Chr1-7). The background lines in gray indicate collinear blocks in the whole rose genome, and the collinear relationships of RCAP2/ERF genes are indicated by solid color lines

contributions to gene expression of the RcAP2/ERF gene. However, RcAP2/ERF gene promoters had a lower abundance of some motifs such as LTR (33.8\%), DREB (32.4\%), and salicylic acid responsiveness (31.6\%), suggesting that these regions controlled the expression of particular genes only under appropriate circumstances. These analyses of cis-regulatory elements implied that $R c A P 2 / E R F$ genes may not only be involved in hormone signaling but also participated in reacting to external stresses.

Isolation, structure, and promoter analysis of RCDREB2B As DREB A2 subgroup members are considerably involved in abiotic stress tolerance in different plant species, we select $R c D R E B 2 B$, which belongs to DREB A2 subgroup member, for further analysis. The primers of $R c D R E B 2 B$ were designed for PCR amplification, and full-length cDNA sequences were cloned. The sequences of $R c D R E B 2 B$ cDNA and deduced amino acid were submitted to the National Center for Biotechnology Information (NCBI) GenBank (MH152409). It was revealed by sequence analysis that an open reading frame (ORF) of $585 \mathrm{bp}$ was contained in the full-length cDNA and encoded a putative protein of 194 amino acids with a $\mathrm{pI}$ of 9.03 and a predicted molecular weight of $21.2 \mathrm{kDa}$.

It is evident from multiple sequence alignment between reported DREB A2 subgroup members (AtDREB2B, TaDREB1, ZmDREB2A, and HvDRF1) and RcDREB2B that all proteins demonstrate the characteristics features of DREB proteins, specifically a conserved DNA-binding domain (AP2-domain) comprising 64 amino acids, and conserved YRG, WLG, and RAYD motifs. Glutamic acid (E19) was conserved at the 19th position in the AP2 domain in RcDREB2B, HvDRF1 and TaDREB1 (Additional file 3: Figure S1). Moreover, the AP2 domain manifested a greater degree of amino acid identity than the N-termini and Ctermini of the proteins. There was also a typical nuclear localization signal (NLS) in the N-terminal region (Additional file 3: Figure S1). The sequence analysis revealed that RcDREB2B possessed a high degree of sequence homology to AtDREB2B (30\% identity) in Arabidopsis thaliana, TaDREB1 (36.49\% identity) in Triticum aestivum, HvDFR1 (26.61\% identity) in Hordeum vulgare, and ZmDREB2A (30.09\% identity) in Zea mays.

Based on the amino acid sequences of RcDREB2B and other plants' DREB proteins, a phylogenetic tree was constructed. Phylogenetic analyses revealed that DREB proteins can be categorized into six sub-groups (A1A6), among which RcDREB2B along with AtDREB2A, AtDREB2B, TaDREB1, ZmDREB2A, and HvDRF1 belong to the A2 sub-group (Fig. 5a). Moreover, RcDREB2B exhibited high similarity to AtDREB2A and AtDREB2B. Therefore, this gene was named RcDREB2B instead. Collectively, the data implies that RcDREB2B is an A2 sub-group member of the DREB subfamily.

\section{Expression, nuclear localization, and transcriptional activity of RcDREB2B}

We analyzed $R C D R E B 2 B$ expression in roots and leaves of the rose seedlings under normal drought stress (ND), medium drought stress (MD), and severe drought stress (SD) by real-time quantitative PCR (RT-qPCR). RcDREB2B was constitutively expressed in leaves tissues under the treatments we tested. In the root tissue, expression levels of $R c D R E B 2 B$ were significantly downregulated under $\mathrm{MD}$ and $\mathrm{SD}$ treatment, in comparison to the ND controls (Fig. 5b). These findings indicated that $R c D R E B 2 B$ might be involved in mediating drought stress signaling transduction.

Investigation of the subcellular localization of RcDREB2B was conducted by constructing GFP and RcDREB2B-GFP and transformed them with Arabidopsis protoplast cells. Figure $5 \mathrm{c}$ shows that GFP fluorescence is equally divided in the nucleus and the cytoplasm with the control plasmid GFP, whereas the RcDREB2B-GFP fusion protein, was only located in the nucleus (Fig. $5 \mathrm{c}$ ). A yeast assay system was used to estimate whether RcDREB2B was capable of activating transcription. Yeast strain Y2HGold was transfected individually with pGBKT7RcDREB2B, pGAL4 (positive control), and pGBKT7 (negative control) plasmids. All transformed yeasts were able to grow in SD/Trp- media. The yeast transfected with pGBKT7-RcDREB2B and pGAL4 was able to grow on the $\mathrm{SD} /$ Trp-His-Ade-, however the negative control was not able to grow. Besides, blue color was observed upon incubation of the yeast extract of yeast transfected with pGBKT7-RcDREB2B and pGAL4 with X-gal (Fig. 5d). The outcome implies that RCDREB2B is a nuclearlocalized transcriptional activator.

\section{Overexpression of RCDREB2B reduced the high salinity tolerance in transgenic Arabidopsis}

Driven by a constitutive super promoter [24], RcDREB2B was overexpressed in Arabidopsis for further functional 


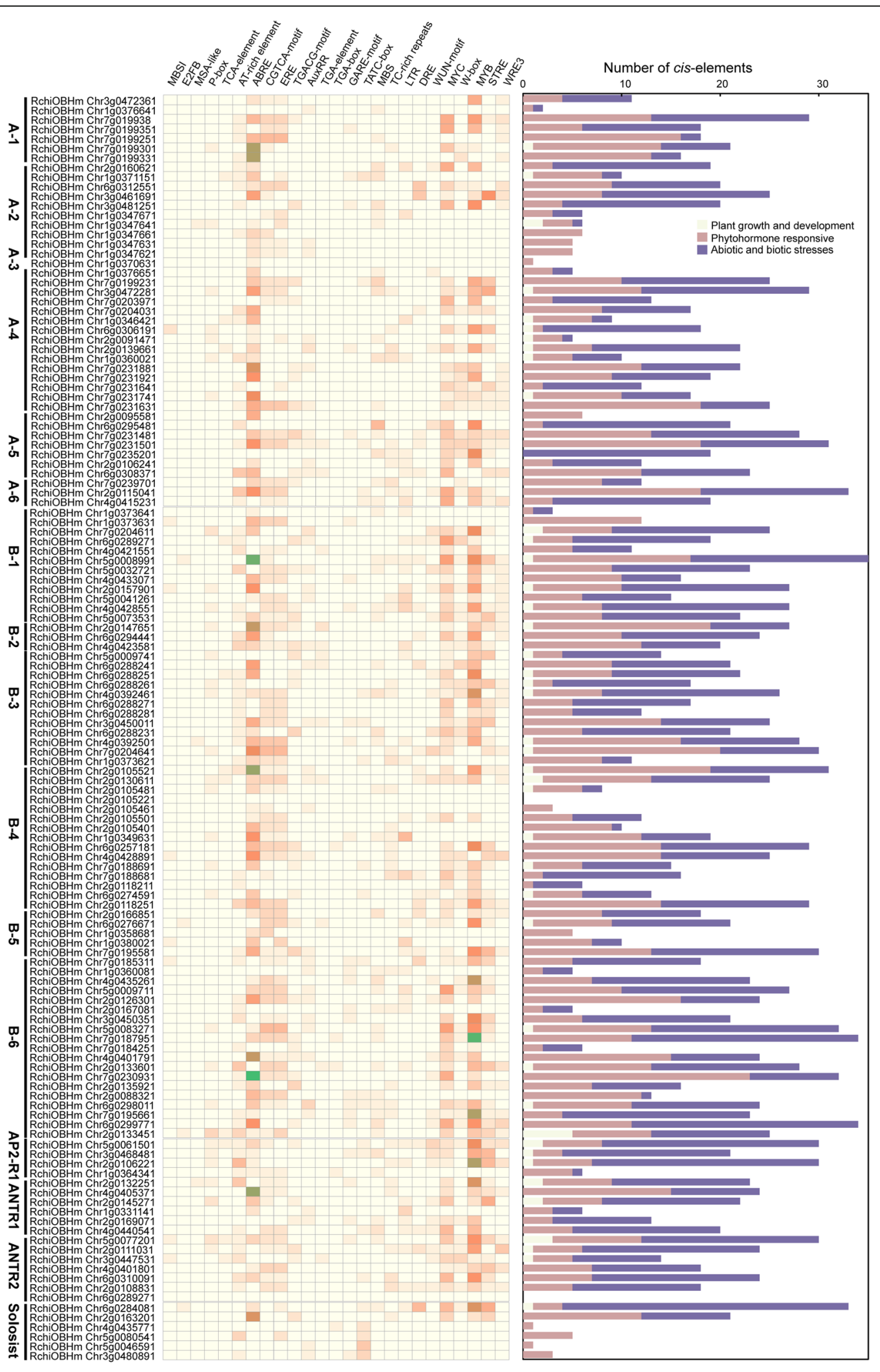

$\begin{array}{lllllll}0 & 3 & 6 & 9 & 12 & 15 & 18\end{array}$

Fig. 4 (See legend on next page.) 
(See figure on previous page.)

Fig. 4 Putative cis-elements in RCAP2/ERF promoters. The promoter region of RCAP2/ERF genes shows cis-elements (1500 bp upstream). The assessment was done using PlantCARE, a nucleotide sequence pattern database for plant cis-acting regulatory DNA components. Symbols show binding sites. The left panel showed the different AP2/ERF subgroup members. Heatmap represented different cis-elements of different RcAP2/ ERFs promoter regions. MBS I, MYB binding site involved in flavonoid biosynthetic genes regulation; E2F, cis-acting element involved in the cell proliferation, differentiation, and endocycle; MSA-like-element, a cell cycle-related element; P-Box, gibberellin-responsive element; TCA-element, cis-acting element involved in salicylic acid responsiveness; AT-rich element, the essential elements of replication origins of bacterial replicons; ABRE, cis-acting element involved in the abscisic acid responsiveness; CGTCA-motif, cis-acting regulatory element involved in the MeJAresponsiveness; TGACG-motif, cis-acting element involved in the abscisic acid responsiveness; AuxRR-core, cis-acting element involved in the auxin responsiveness; TGA-element, auxin-responsive element; TATC-box, gibberellin-responsive element; MBS, MYB binding site involved in droughtinducibility; TC-rich repeats, cis-acting element involved in defense and stress responsiveness; LTR, cis-acting element involved in low-temperature responsiveness; DRE, cis-acting element involved in dehydration, high salinity, or low temperature; WUN-motif, wound-responsive element; MYC, cis-acting element involved in the dehydration and abscisic acid responsiveness; W box, WRKY binding site; MYB, cis-acting element involved in the dehydration and abscisic acid responsiveness; STRE-element, stress-responsive element; WRE3-element, cis-acting regulatory element involved in high temperature. Three different types of cis-acting elements (plant growth and development, phytohormone responses, and abiotic and biotic stresses) are represented by different colors, as shown on the right

characterization. The T3 generation of three independent RcDREB2B-overexpression lines (OE\#4, 5, and 16) with different expression levels was subsequently chosen for analysis (Fig. 6a). No apparent morphological changes in terms of flower diameter, petal length, petal width, and single petal area were observed between $\mathrm{VC}$ and RcDREB2B-OE plants (Additional file 4: Figure S2). MS media with $0,50,100,150$, and $200 \mathrm{mM} \mathrm{NaCl}$ were used to sow seeds of the RcDREB2B-OE and VC (Fig. $6 b)$. No significant morphological differences were observed in the rate of seed germination between RCDREB $2 B-\mathrm{OE}$ and control lines showed when grown on 0,50 , and $100 \mathrm{mM} \mathrm{NaCl}$ plates. However, when supplemented with 150 and $200 \mathrm{mM} \mathrm{NaCl}$, the germination rate of all plants reduced, accordingly. Upon supplementation with $150 \mathrm{mM} \mathrm{NaCl}$, the germination rates of OE\# 4,5 , and 16 were $52.8,54.1$, and $16.2 \%$, respectively, significantly lower than VC controls (91.7\%) (Fig. 6b, c).

To further analyze the root phenotypes of RcDREB2B-OE plants under salt stress, we placed 6day-old seedlings in the MS media plates containing $0,100,150$, and $200 \mathrm{mM} \mathrm{NaCl}$, and treated for 10 days (Fig. 6d). The increment root length between RcDREB2B-OE and VC exhibited no significant variation when grown on 0 and $200 \mathrm{mM} \mathrm{NaCl}$ plates. But, at $\mathrm{NaCl}$ concentration of 100 , or $150 \mathrm{mM}$, the $\mathrm{VC}$ displayed a significantly longer increment of root length than RcDREB2B-OE lines (Fig. 6e). For instance, when the growth was carried out on MS plates containing $150 \mathrm{mM} \mathrm{NaCl}$, the increment in root length of the VC plants was $0.88 \mathrm{~cm}$, while the increment in root

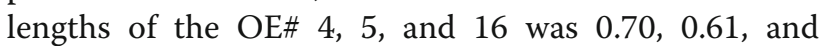
$0.70 \mathrm{~cm}$, respectively (Fig. 6e). Without $\mathrm{NaCl}$ treatment, there were no significant differences between RcDREB2B-OE lines and VC controls in terms of lateral root number. However, the lateral root number the same in RCDREB2B-OE lines was significantly more than $\mathrm{VC}$ in plants when exposed to the 150 and $200 \mathrm{mM} \mathrm{NaCl}$ condition (Fig. 6d, f).
Next, two distinct histochemical staining assays were carried out on Arabidopsis seedlings to detect $\mathrm{O}_{2}{ }^{-}$and $\mathrm{H}_{2} \mathrm{O}_{2}$ using DAB and NBT, respectively. Ten-day-old seedlings of RcDREB2B transgenic and VC plants were exposed to $200 \mathrm{mM} \mathrm{NaCl}$ or water (CK) solutions for 1 h. No significant differences in histochemical staining of seedlings leaves and roots were observed in both RcDREB2B-OE and VC plants under CK conditions (Fig. $6 \mathrm{~g})$. Compared with VC, RcDREB2B-OE lines showed significantly stronger blue and brown color under 200 $\mathrm{mM} \mathrm{NaCl}$. Quantities analyses of $\mathrm{H}_{2} \mathrm{O}_{2}$ and $\mathrm{O}_{2}{ }^{-}$indicated $\mathrm{RcDREB} 2 \mathrm{~B}-\mathrm{OE}$ exhibited significantly higher content than VC controls (Additional file 5: Figure S3). The results demonstrate that $R C D R E B 2 B-\mathrm{OE}$ accumulated higher $\mathrm{H}_{2} \mathrm{O}_{2}$ and $\mathrm{O}_{2}{ }^{-}$levels compared with $\mathrm{VC}$ plants under salt stress, thus implying that $R c D R E B 2 B$ plays a negative role in controlling reactive oxygen species. These results demonstrate that overexpressing RCDREB2B in Arabidopsis decreased salt tolerance during the seedling stage as well as germination.

\section{Overexpression of RCDREB2B leads to ABA sensitivity in Arabidopsis}

To elucidate the involvement of RcDREB2B in ABA signaling, we conducted a phenotypic analysis of RcDREB2B-OE plants at the germination and root development stages in response to ABA (Fig. 7). Seeds were sown on MS medium supplemented with different concentrations of $\mathrm{ABA}(0,1.2$, and $1.6 \mu \mathrm{M})$ for 9 days (Fig. $7 \mathrm{a})$. In the absence of $\mathrm{ABA}$, the germination rates of RCDREB2B-OE and VC plants were $100 \%$. However, with $1.2 \mu \mathrm{M}$ ABA, the germination rates decreased to $70.05-77.22 \%$ for RcDREB2B-OE lines, and $91.98 \%$ for VC. Similar inhibition was observed in these plants grown on a $1.6 \mu \mathrm{M}$ ABA plate, the germination rates of OE\#4, OE\#5, and OE\#16 were $37.4,64.1$, and $61.5 \%$, respectively, whereas VC germination rate was $90.0 \%$ (Fig. $7 \mathrm{~b})$. We also tested the root phenotype of RcDREB2B$\mathrm{OE}$ and $\mathrm{VC}$ exposed to 0 and $100 \mu \mathrm{M} \mathrm{ABA}$ (Fig. 7c). 

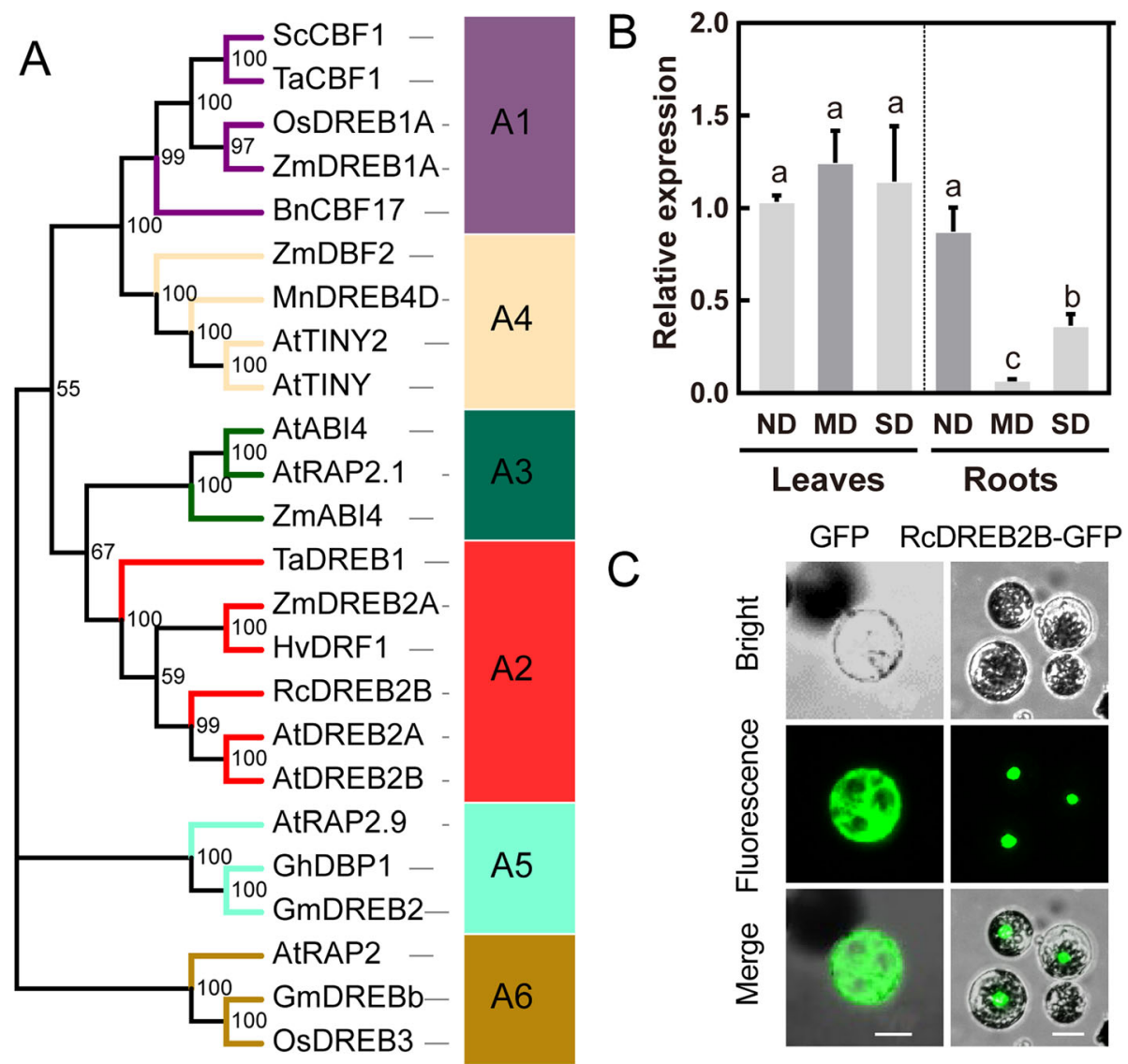

C

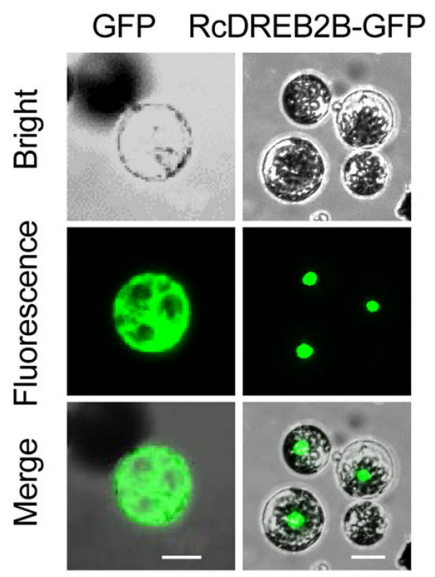

$\mathrm{D}$

SD/Trp- SD/Trp-His- $\quad$ SD/Trp-His-X-gal

$10^{0}$
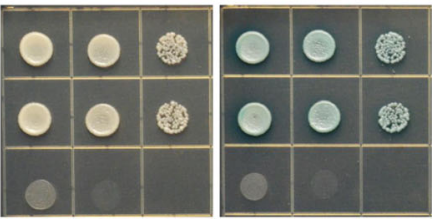

Fig. 5 Characteristics of RCDREB2B. a Phylogenetic tree of RCDREB2B and other plant DREB proteins. The phylogenetic tree was constructed using Evolview and MEGA 7.0 with bootstrap values of 1000 replicates. The accession numbers of the protein sequences that have been retrieved from NCBI and species designations are listed below: Arabidopsis thaliana: AtDREB2A (AB007790), AtDREB2B (NM_111939.2), AtABI4(A0MES8), AtTINY2 (AY940160.1), AtTINY(Q39127), AtRAP2.1 (Q8LC30), AtRAP2.9 (NM_179009.1), AtRAP2 (AAP04063.1); Oryza sativa: OsDREB1A (AF300970), OsDREB3 (NP_001048142); Glycine max: GmDREB2 (ABB36645), GmDREBb (AAQ57226); Gossypium hirsutum: GhDBP1(AAO43165.1); Zea mays: ZmDREB1A (AF450481), ZmDREB2A (NP_001105876), ZmABI4 (AY125490), ZmDBF2 (AF493799); Hordeum vulgare: HvDRF1 (AY223807); Triticum aestivum: TaDREB1 (DQ195068), TaCBF1 (AF376136); Secale cereal: ScCBF1 (AF370730); Brassica napus: BnCBF17 (AF499034); Morus notabilis: MnDREB4D (AHJ25980.1). Different color lines indicate different subgroups of the DREB subfamily. $\mathbf{b}$ Transcript levels of RCDREB2B. The expression levels of RCDREB2B in leaves and roots during no drought (ND), mild drought (MD), and severe drought (SD) treatments. The mean fold changes of $R C D R E B 2 B$ in $\mathrm{MD}$ and SD were compared with ND. We present results as means $\pm \mathrm{SD}$. $n=3$. Different letters denote significant differences at $P<$ 0.05 with one-way ANOVA analysis. c Subcellular localization of RcDREB2B in Arabidopsis protoplast cells. RcDREB2B-GFP fusion protein or GFP alone is expressed under the control of pCAMBIA 1300 in Arabidopsis protoplast cells. The photographs were taken in the green channel, bright channel, and merge channel. Bars $=20 \mu \mathrm{m}$. $\mathbf{d}$ Transcriptional activity analysis of RcDREB2B. The full-length ORF of RcDREB2B was fused to the GAL4 DNA-binding domain in the vector pGBKT7 to generate pGBKT7-RCDREB2B, and the construct was converted into yeast strain Y2HGold. Yeast dilutions were grown on SD medium lacking Trp, and the same medium containing with or without X-gal but lacking with Trp, His, Ade. PGBKT7 and PGAL4 plasmids were respectively used as negative and positive control 


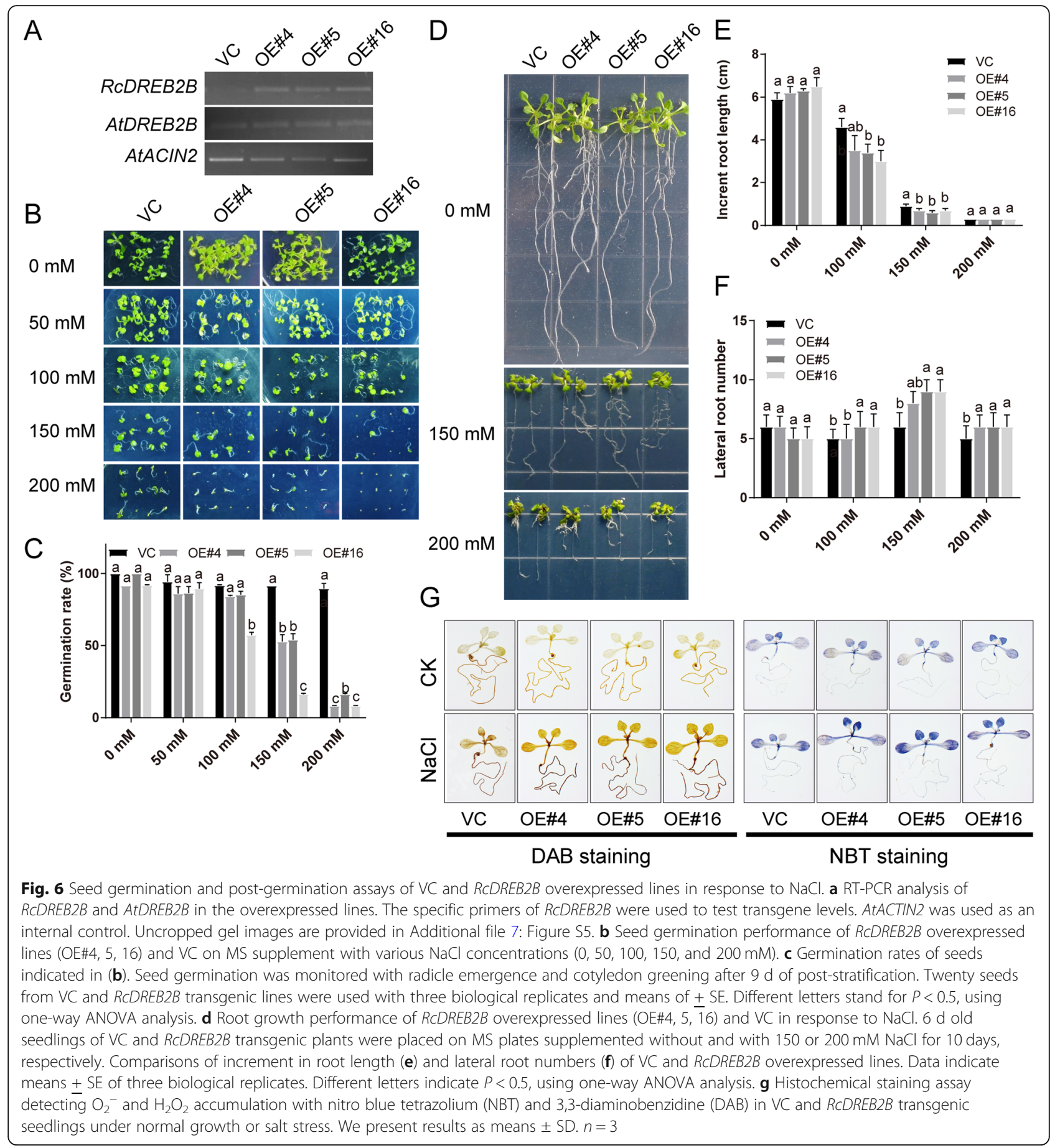

However, no significant differences were observed in the root phenotype of RcDREB2B-OE and $\mathrm{VC}$ seedlings in the absence of ABA. As shown in Fig. $7 \mathrm{~d}$, the RcDREB2B-OE plants exhibited a $0.97-1.20 \mathrm{~cm}$ increment in root length under $100 \mu \mathrm{M}$ ABA in comparison to $2.4 \mathrm{~cm}$ in VC controls. RcDREB2B transgenic plants had a significantly lesser lateral root number as compared to $\mathrm{VC}$ in the presence of $100 \mu \mathrm{M} \mathrm{ABA}$ (Fig. 7e). Such results suggest that RCDREB2B causes enhancement in ABA sensitivity of plants during germination and thereafter.

Overexpression of RCDREB2B exhibit sensitivity to inhibition of germination by osmotic stress in transgenic Arabidopsis

Since the sensitivity of plants towards $A B A$ at the seed germination stage of RcDREB2B-OE has been increased, we predicted that overexpression of $R c D R E B 2 B$ may also 

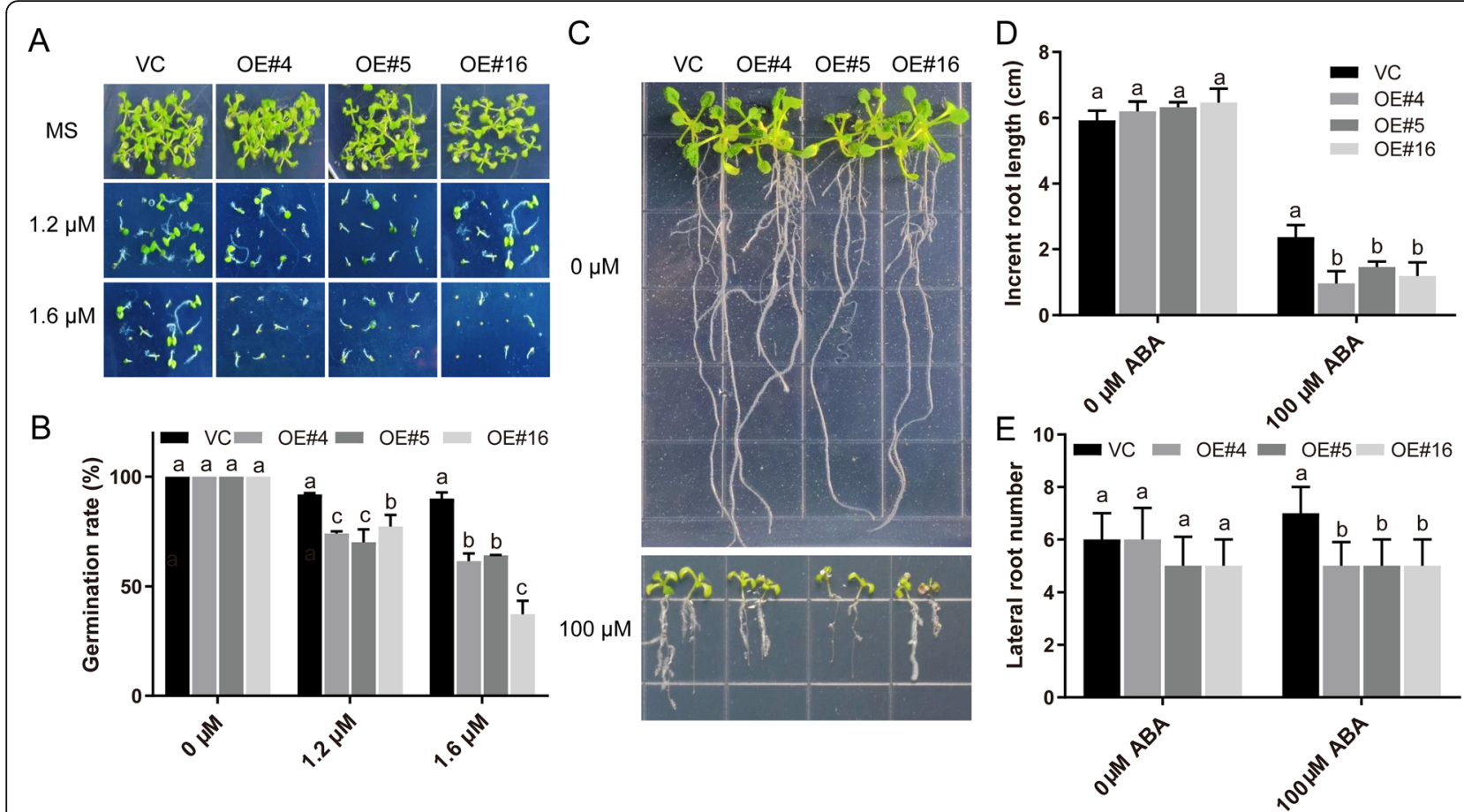

Fig. 7 Germination and root growth analysis of $V C$ and $R C D R E B 2 B$ overexpressed lines in response to $A B A$. a Seed germination performance of $V C$ and RCDREB2B overexpressed lines on MS with different ABA concentrations $(0,1.2 \mu \mathrm{M}, 1.6 \mu \mathrm{M})$ after 9 days. $\mathbf{b}$ Statistical analyses of germination rates are indicated in (a). Seed germination rates were measured after $9 \mathrm{~d}$ growth. $\mathbf{c}$ Root growth of VC and transgenic seedlings on MS agar plates containing $0 \mu \mathrm{M}$ or $100 \mu \mathrm{M}$ ABA. Representative photos were taken following 10 days of the transfer. Seedlings were 6-days-old at the time of transfer. Increment in root length (d), and lateral root number (e) of $V C$ and RCDREB2B transgenic plants in response to ABA. Different letters indicate significant differences in three independent experiments at $P<0.05$ using one-way ANOVA analysis

influence plant tolerance to stress. In an attempt to investigate this, the seed germination of RcDREB2B-OE and VC controls under $0,4,8,12$, and $16 \%$ PEG treatment was analyzed (Additional file 6: Figure S4). RcDREB2B-OE and VC lines when grown on 0,4 , and $8 \%$ PEG plates showed no significant differences. The germination rate of RcDREB2B$\mathrm{OE}$ and VC controls were significantly inhibited by 12 and $16 \%$ PEG, but the inhibition in the VC was less severe. When supplemented with $12 \%$ PEG, VC germination rates declined to $\sim 92.28 \%$, whereas $R c D R E B 2 B-O E$ lines displayed 75.6-78.6\% germination (Additional file 6: Figure S4). Furthermore, RcDREB2B-OE lines displayed a 67.5$71.7 \%$ germination ratio in MS medium supplement with 16\% PEG whereas VC germination was reduced to $93.0 \%$ (Additional file 6: Figure S4). It is evident from these results that $R c D R E B 2 B-O E$ seeds display a sensitivity to inhibition of germination upon stress due to osmosis.

\section{Overexpression of RCDREB2B in Arabidopsis altered the expression of osmotic and $A B A$-responsive genes}

To further explore the molecular mechanisms through which $R c D R E B 2 B$ controls the sensitivity of germination, RT-qPCR analysis was carried out to examine the expression pattern of four osmotic stress-responsive genes (AtDREB2A [25], AtWRKY33 [26], and AtERF5 [27],
AtKIN1 [28]), seven ABA-responsive genes (AtABI2 [29], AtKAI2 [30], AtADH1 [31], AtCHS [32], AtABF3 [33], AtABF4 [34], and AtICK1 [35]), one ABA biosynthesis gene (AtNCED3 [36]) in RcDREB2B-OE and VC plants under normal growth condition. Relative expression of AtDREB2A, AtWRKY33, AtERF5, AtKINI, AtABI2, AtKAI2, AtADH1, and AtNCED3 was significantly increased in the RCDREB2B-OE plants under normal conditions compared with VC controls. Besides, the chalcone synthase gene $C H S$ [32], which is classified as the stress-responsive biosynthetic genes, was upregulated. But, two abscisic acid-responsive elementbinding factors (AtABF3, AtABF4) exhibited a significant reduction under normal conditions (Fig. 8). The enhanced or attenuated expression of these genes in RcDREB2B might contribute to the ABA-induced sensitivity to inhibition of germination as well as salinity and drought stress during the period of seed germination and early seedling development.

\section{Discussion}

Studies of TFs have been evolved to become an integral element in functional genomics research. TFs, with the involvement of various genes, mediate stress responses by inducing activating or inhibitory signaling pathways. 

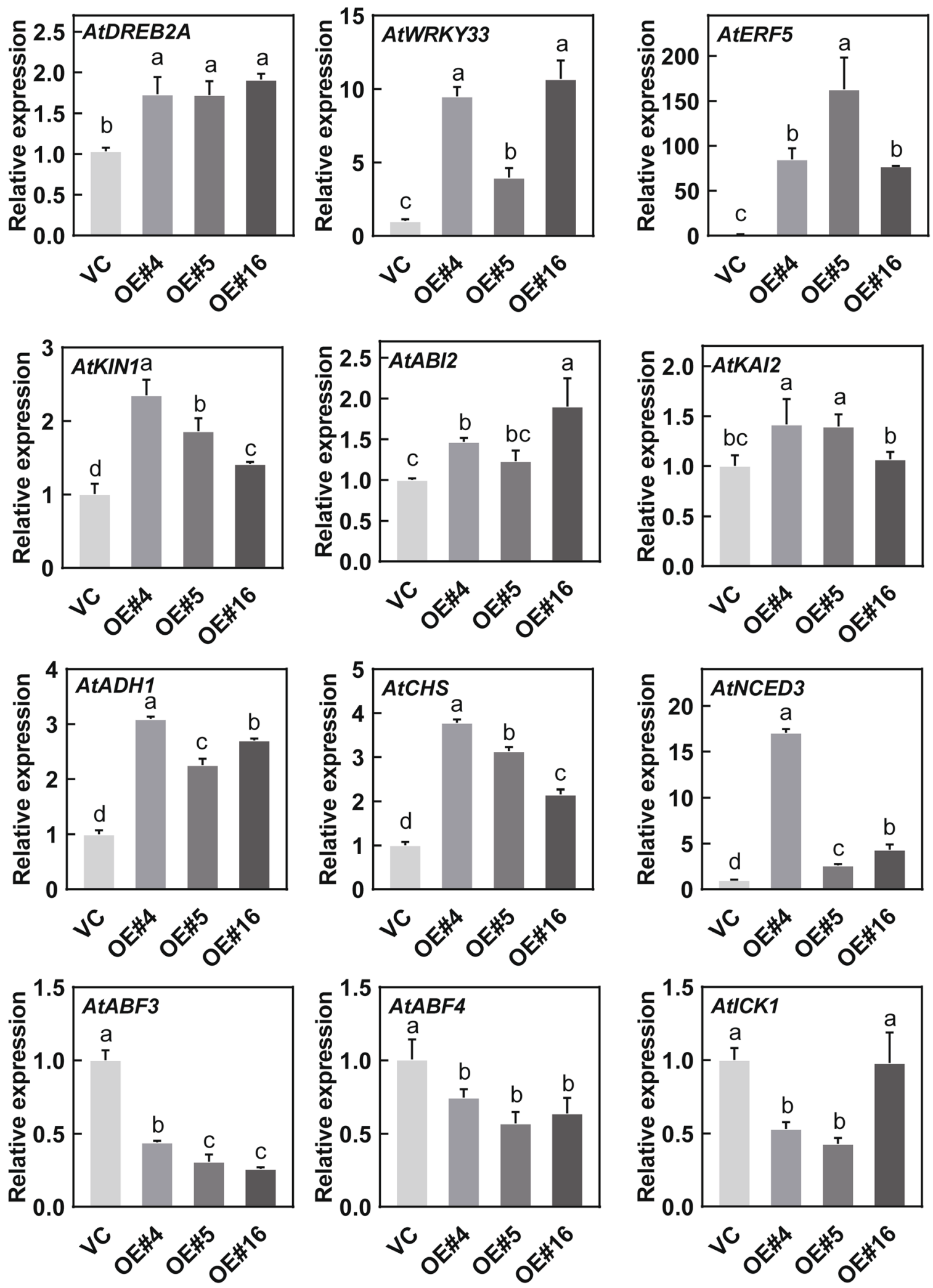

Fig. 8 (See legend on next page.) 
(See figure on previous page.)

Fig. 8 Expression analysis of osmotic and ABA-related genes in VC and RCDREB2B overexpressed lines. Analysis of the osmotic stress-responsive genes and ABA-related genes in VC and RCDREB2B transgenic lines was performed using RT-qPCR. Total RNA extraction was carried out from 3week-old seedlings grown under normal growth conditions. Three separate replicates were carried out for RT-qPCR analysis. The relative expression of individual genes of VC was set to 1.0, and AtACTIN2 was used as an internal control gene. An additional file (Additional file 2: Table S2) lists the primers used. Different letters indicate significant differences in three independent experiments at $P<0.05$ using one-way ANOVA analysis

Plants, thereby, make use of these mechanisms to adapt to different environmental conditions [37]. Among plant TFs, the AP2/ERF family is one of the largest concerning the size. AP2 is known to be involved in plant reproduction and homeostasis particularly in plants' responses to biological or non-biological stresses [8]. Genome-wide scan of AP2 has been systematically carried out in Arabidopsis [5], Fagopyum tataricum [19], Setaria italic [38], Vitis vinifera [39], peach (Prunus persica) [40], castor bean (Ricinus communis) [41], and Salix arbutifolia [42]. Despite this, the information on the AP2/ERF genes of rose is still scant. Herein, we identified and investigated the information regarding AP2/ ERFs in rose. Our study revealed, that a total of 135 AP2/ERFs were identified, including 44 DREB, 68 ERF, 17 AP2, and 6 Solosist subfamily members, possessing identical structural features with other plants. However, in different species, the numbers of AP2/ERFs varied largely. Arabidopsis contains 167 AP2/ERF genes, $V$. vinifera contains 132 AP2/ERF genes, $F$. tataricum contains 135 AP2/ERF genes, and peach contains 130 members. The genome of these plants varies greatly in terms of size: rose $(600 \mathrm{Mb}), V$. vinifera $(475 \mathrm{Mb})$, peach $(265 \mathrm{Mb})$, and F. tataricum $(489 \mathrm{Mb})$, which shows stable AP2/ERF superfamily member numbers with no direct relation to genome size.

Analysis of domain conservation and gene sequences revealed pattern conservation in RcAP2/ERF genes from each group. The preserved patterns and intron-exon distribution of RcAP2/ERFs were analyzed to obtain a better understanding of the structural features of rose AP2/ ERFs. While introns were absent in $68.38 \%$ of RcAP2/ ERF, AP2 subgroup genes had between 1 and 9 introns. The conserved motif analysis of RcAP2/ERFs supported the indicated phylogenetic relationship and classification of rose AP2/ERFs. Rose AP2 gene composition was found to resemble that of F. tataricum [19] and Hordeum vulgare [43]. AP2 structural subgroup gene differences suggested the presence of numerous evolutionary gene changes. Conserved TF motifs played an important role in the work of particular groups, as recognized in DNA binding, transcriptional behavior, and protein interaction [37]. The examination of motifs demonstrated that the majority of rose AP2/DREB subfamily genes expressed motifs 1, 7, 8, and 10 (Fig. 1). Motifs 1, 2, 3, and 7 have been found in different ERF system groups, suggesting that they have valuable functions associated with them. Results have shown that, while several variations of the AP2/ERF family genes have been significantly conserved, emerging evolutionary motifs that carry out novel activities in some plants need more research. Two patterns were found relatable to the AP2 domain, that is, motifs 1 and 2. Motif 1 covered the largest area of the AP2 domain (PF00847), even Gx4E, WLG, and AYD components. While motif 2 retained the AYD group. All RcAP2/ERFs bore a minimum of one of the two patterns that exhibit high conservation of the AP2 domain in genes of RcAP2/ERF. Besides the AP2 domain-related motifs, there were another eight motifs outside the AP2 domain in the group-specific distributions. Motif 8 was expressed by members of group A4 of the DREB subfamily, distinguished by four chains of preserved amino acid residues: LNFP, D [IV] QAA / DIR [RA], and LPRP (Fig. 1). Preserved amino acids have been identified as critical sites in Arabidopsis for serine / threonine-proteins kinase-12 that binds CBL [44], dehydration-responsive item binding protein- $1 C$ and protein-G [45], ARF19 [45], disease resistance [46], and ERF037 [44], respectively. Nevertheless, the roles of other motifs are still unclear, and an extensive amount of investigation is required to understand their biological functions.

Previous studies have reported gene duplication as a major source of evolution of the gene family, including individual gene duplications, segmental duplication, tandem duplication, chromosomal fragments, transposition events, and entire genomes duplication [47]. Furthermore, segmental duplication and translocation have enabled plants to rapidly adapt to any change in the environments [48]. To acquire a better understanding of the expansion mechanism of AP2 genes, we carried out an analysis of the gene duplication events in rose. We identified at least nine pairs of $R c A P 2 / E R F$, including three tandem duplications and two segmental duplications. The RcAP2/ERF genes are randomly and unevenly distributed on 7 chromosomes. Segmental duplication events were also detected in each chromosome and accounted for $20 \%$ of the RcAP2/ERF. Our findings have shown that gene replication influences RcAP2/ERF chromosomal position and gene family extension depends on sequence duplication, either of the tandem or segmental kind. 
Gene expression specificity depends on cis-regulatory elements and their associations with TFs [49]. Fifteen cisacting regulatory elements were found in RcAP2/ERF roses, including ABRE, LTR, MYB, MYC, and DRE, and were designated to $1.5 \mathrm{~kb}$ upstream of the RcAP2/ERF initiation codon. These cis-elements are active in the control of genes under abiotic or biotic stresses. The ABRE element class binds to ABA-dependent TFs which are conservatively strong. Their promoters also contain stressresistant genes such as those related to dehydration, drought, salt, and low temperature. In response to nonbiological stress, they regulate the expression of associated genes [50]. Cis-acting ABRE regulatory elements are present in most upstream regulatory sequences of RcAP2/ $E R F$, suggesting that these genes play an indispensable part in reacting to environmental stimuli. The LTR element which mediates the response to low temperatures is also cis-acting [51]. RchiOBHmchrlg0331141 and RchiOBHmchrlg0349631 possess three continuous repetitive LTRs, suggesting that these two genes can operate under low-temperature responses. MYB cis-element is the DNA binding site for MYB transcription factors and regulates defense, abiotic and biotic stress reactions, differentiation, development, metabolism, etc. [52]. Within the RchiOBHmchr3g0449251 promoter regions, many repeated MYB cis-elements have been identified, indicating that RchiOBHmchr3g0449251 is a putative direct target of MYB transcription factors. Most RcAP2/ERF carries one or more W-BOX cis-elements, where DNA binding occurs for WRKY transcription factors. They are also involved in the genetic control of plant reproduction and homeostasis, leaf aging, cell signaling, and many abiotic and biotic stress reactions [53]. When the cis-regulatory elements were examined they showed, that the promoter sequences of the RCAP2/ERF genes were not uniformly distributed. MYB and MYC cis-regulatory zones were most abundant in RcAP2/ERF promoters, thus implying that RcAP2/ERFs may be regulated by other TFs. A relatively large number of cis-regulatory elements share homology with TFs and mediate hormonal signaling or abiotic stress tolerance. This indicates that RcAP2/ERF TF regulation is integral to the growth and stress responses of the rose.

DREB proteins comprise one of the largest group of AP2/ERF TFs and are widely detected in several plants, such as rice (Oryza satviva) OsDREB2A [54], soybean (Glycine max) GmDREB2 [55], maize (Zea mays) ZmDREB2.7 [56], tomato (Solanum lycopersicum) SIDREB2 [57], as well as some desert plants, such as Eremosparton songoricum DREB2B [58], Caragana korshinskiis DREB1 [59] and Tamarix hispida DREB [60]. These DREB proteins are known to play key roles in response to abiotic stresses, particularly for A1 and A2 DREB subgroup members. In the present study, we chose an DREB A2 subgroup member, RchiOBHmChr2g0160621, and named it RcDREB2B, which has high homology with AtDREB2A and AtDREB2B (Fig. 5a). Both of these two genes are induced by dehydration and high-salt stress, and AtDREB2A is one of the most common TF in regulating drought-responsive gene expression through regulating stress-inducible genes that contain DRE sequences [7]. Our results showed that $R c D R E B 2 B$ expression was significantly suppressed, especially for MD in the root samples, under drought stress treatments, (Fig. 5b). The expression pattern of $R C D R E B 2 B$ in response to drought stress does not corroborate with other reported DREB2B members, suggesting the possible occurrence of diverse functions among different plant species. Also, the regulation analysis conducted for the expression of RcDREB2B under the treatments we tested, should further be investigated.

Many studies have reported that DREB gene products being involved in abiotic stress responses and enhancement of plant tolerance towards abiotic stress due to overexpression of DREB genes [5]. The current study shows that overexpression of RcDREB2B in Arabidopsis resulted in enhanced sensitivity to high salt, ABA, PEG at the germination and seedling stage, together with a less increment in root length. Similar results have been observed with transgenic rice overexpressing physic nut (Jatropha curcas) JcDREB2 gene [61], which caused increased sensitivity to salt stress. Until now, the mechanism regulating the function of DREB2-type genes under abiotic stress is not well-studied. 26S proteasome often degrades DREB2 protein stability under non-stress conditions, through its negative regulation domain [62]. AtDREB2A was ubiquitinated by DREB2A-INTERACTING PROTEINS abbreviated as DRIP1 and DRIP2 in the nucleus [62]. Ubiquitination presumably leads to its subsequent DREB2 type protein degradation, thus negatively regulating the response to drought. Detail evidence needs to be illustrated not only transcriptional regulation but post-translational modification like ubiquitination or phosphorylation, and these may be a suitable conserved mechanistic pathway for the degradation or activation of DREB2 type proteins under stress conditions.

The role of ABA in many plant processes such as dormancy and formation of seeds, growth arrest and inhibition of germination in the early seedling stage under unfavorable environmental conditions has been well established [63]. Our study suggested that RcDREB2B expression in Arabidopsis restricted seed germination and seedling growth by upregulating the expression of ABA-regulated TF AtKAI2 (Fig. 8). This gene has been shown to play a key role in ABA inhibition during the germination of seeds and the early development of seedlings [63]. AtKAI2 upregulation causes the ABA allergic 
phenotype of $R C D R E B 2 B$ during seed germination and early seedling development. This activation may have caused the ABA accumulation to increase under salt or drought conditions and caused the plants to exhibit the concurrent salt and drought sensitivity, leading to enhanced seed sensitivity. RcDREB2B-OE plants exhibited hypersensitivity to PEG at the germination stage, implying that RcDREB2B regulates osmotic stress tolerance via ABA-mediated cell signaling. In addition, $\mathrm{ABF} / 4$ exhibited less expression level in RcDREB2B-OE lines than VC controls. Depressed expression levels of ABF3/4 may reflect a negative feedback loop needed to dampen the increased ABA sensitivity during germination and post-germination stage in RcDREB2B transgenic plants. These results are consistent with previous studies on some DREB TFs, which possess the ability to be activated by ABA and mediate their downstream gene expression enabling a plant to survive any stressful environment.

\section{Conclusions}

To summarize, in our investigation, we identified 135 AP2/ERF TFs in the rose genome. Their classification and evolutionary relationships were elaborated using a phylogenic, conserved motif, and gene structure analysis. Cis-acting elements of these AP2/ERF TFs revealed that these genes mediate development and stress responses in rose plants. The AP2/ERF family bioinformatics analysis results provide primary resources to investigate the molecular regulation of rose species. Besides, we characterized a novel rose AP2/ERF gene RcDREB2B, the expression of which was repressed by drought. RCDREB2Boverexpressing transgenic Arabidopsis enhanced sensitivity to salt and drought stress and exhibited an ABAhypersensitive phenotype. The outcome of this investigation will help to increase our understanding of the roles played by RcDREB2B in a rose in responses to abiotic stress.

\section{Methods}

\section{Sequence identification and examination of Rosa} chinensis RcAP2/ERF genes

We conducted a Hidden Markov Model (HMM) through Pfam's (http://pfam.sanger.ac.uk) RcAP2/ERF amino acid sequences [40] to check whether an AP2 domain (PF00847) was present in the Rosaceae database of genomes (https:/www.roseceae.org/). Amino acid composition, chemical, and physical characteristics of RCAP2/ERF TFs were examined using the ProtParam method (https://web.expasy.org/protparam/) [64]. WoLF PSORT (https://wolfpsort.hgc.jp/) [65] was used to make predictions regarding the subcellular localization of proteins inside cells.
Gene structure, chromosomal localizations, conserved motif, phylogenic, and promoter analyses

The default settings of ClustalW facilitated the investigation of the RcAP2/ERF structure [66]. The Gene Display Structure Server (http://gsds.gao-lab.org/) was used to evaluate the components of the RcAP2/ERF introns and exons. The MEME tool (http://meme-suite.org/tools/ meme) was employed to predict and analyze the motifs of $R c A P 2 / E R F$ proteins, with parameters set as follows: motif width 6-200, the maximum number of motifs: 10 and default values were used for the remaining parameters [67]. RcAP2/ERF TFs were mapped to rose chromosomes according to the $R c A P 2 / E R F$ location presented by Mapchart v2.2 software and the Circos tool [68]. AP2/ERF proteins of apple (Malus domestica) and Arabidopsis were obtained from the plant transcription factor database (http://plntfdb.bio.uni-potsdam.de/v3.0/). The phylogenetic relationship of RcAP2/ERF was established by the neighbor-joining method with 1000 bootstraps values and was then visualized with the help of MEGA 7.0 software [69]. The evolutionary background was derived using an online tool of Evolview [70]. $R c A P 2 / E R F$ genes promoter regions, the $1.5 \mathrm{~kb}$ sequence upstream of initiation codons of each gene were analyzed and annotated in Plant cis-acting Regulatory DNA Elements (PlantCARE) [71].

\section{Plant growth and drought stress treatment}

Rose (Rosa chinensis 'Mutabilis') plant seeds were harvested at Qingdao Agricultural University trail garden where the annual average temperature was $12.7^{\circ} \mathrm{C}$, however, the annual average sunshine hours were $2622.3 \mathrm{~h}$. After 2 months' verification, planter boxed seedlings grew in media (peat/vermiculite $=1: 1$ ) for 45 days at $23 \pm 1{ }^{\circ} \mathrm{C}$ under a photoperiod comprising $16 \mathrm{~h}$ of light and $8 \mathrm{~h}$ of dark. Then, the seedlings were exposed to drought stress for mild drought stress (MD) for 5 days and severe drought stress (SD) for 10 days. The controls were maintained with water irrigation under the same growth conditions. Three plants were included in each treatment and the leaves and roots were collected at the indicated time points with three biological replicates.

\section{RNA isolation and quantitative reverse transcription PCR analysis}

Total RNA was extracted from rose leaves and roots utilizing the RNA Easy Fast Kit (Tiangen, Beijing, China). Sample RNA in Arabidopsis isolated from leaves of 3-week-old seedlings from the RcDREB2B transgenic lines (OE \#4, OE \#5, and $\mathrm{OE} \mathrm{\# 16)} \mathrm{and} \mathrm{control} \mathrm{(VC)}$ plants. After the validation of RNA concentrations using Nanodrop $^{\mathrm{Tm}} 2000$ (Thermo Fisher Scientific, USA), firststrand cDNA synthesis with $1 \mu \mathrm{g}$ RNA was performed 
using the PrimeScript first-strand cDNA synthesis equipment (TaKaRa, Dalian, China).

For evaluating the rate of gene transcription, RT-qPCR was conducted with SYBR Premix Ex Taq (TaKaRa, Dalian, China). A reaction volume of $20 \mu \mathrm{l}$ was used which comprised: RNase-free water $(8.2 \mu \mathrm{l})$, template (with 5fold dilutions of cDNA) $(1 \mu \mathrm{l})$, each primer $(0.4 \mu \mathrm{l})$, and $2 \times$ SYBR Premix $(10 \mu \mathrm{l})$. The RT-qPCR protocol involved: $30 \mathrm{~s}$ at $95^{\circ} \mathrm{C} ; 95^{\circ} \mathrm{C}$ for $40 \times 5 \mathrm{~s}$ cycles and $34 \mathrm{~s}$ at $60^{\circ} \mathrm{C}$ with a concluding step of $15 \mathrm{~s}$ at $95^{\circ} \mathrm{C}$. RcUBII and AtACTIN2 were used as internal controls in rose and Arabidopsis, respectively. Calculation of the expression level was conducted, using $2^{-\Delta \Delta t}$ as described by Livak and Schmittgen [72]. Three separate replicates were carried out for RT-qPCR. Primers are listed in Table S2.

\section{Amplification and sequence analysis of RCDREB2B}

The amplification of RCDREB2B primers was designed according to the full-length coding sequence of RchiOBHmChr2g0160621 and was used to amplify the cDNA sequence in rose leaves under MD treatment. For sequencing, the products were purified and cloned into the pMD18-T vector (TakaRa, Dalian, China). The molecular weight and isoelectric point (pI) of RcDREB2B were estimated with ExPASy (http://expasy.org/tools/pi tool.html) for computation. DNAMAN software was used to perform multiple sequence alignment. The neighbor-joining method was used to generate phylogenetic analysis and a display was made using Evolview [70] and MEGA7.0 [69] in the light of the similarity of amino acid sequences.

\section{Subcellular localization of RcDREB2B}

Overlapping the PCR method was used to link the open reading frame (ORF) of RcDREB2B without the stop codon, and Sal I/Spel I was used to digesting the recombinant fragment which was then inserted into the corresponding sites of the pCAMBIA 1300-GFP vector [24] to generate pCAMBIA 1300-RcDREB2B-GFP. After verification by sequencing, the construct of pCAMBIA 1300-RcDREB2B-GFP and pCAMBIA 1300-GFP control was converted into Arabidopsis (Columbia) mesophyll protoplast using the PEG-calcium transfection method [73]. A laser scanning confocal microscope (TCS SP5, Leica, Germany) was employed to observe green fluorescent protein signals of the protoplast cells.

\section{Transactivation analysis in yeast}

The pGBKT7-RcDREB2B construct was generated by inserting the full-length sequence of RcDREB2B into the Nde I and EcoR I sites of pGBKT7. The pGAL4 plasmid (positive control), pGBKT7 vector (negative control), and pGBKT7-RcDREB2B recombinant plasmid were then transmitted into $\mathrm{Y} 2 \mathrm{H}$ Gold, the yeast strain that had the reporter genes His3 and LacZ. Transformed cells were verified using PCR followed by plating on SD/-Trp or $\mathrm{SD} /-\mathrm{Trp}$-His-Ade medium. In the following step, the plates were incubated at $30^{\circ} \mathrm{C}$ for $3 \mathrm{~d}$, and the resulting clones were used in a 5-bromo-4-chloro-3-indolylb-Dgalacto-pyranoside (X-gal) assay to examine the transactivation ability of RcDREB2B.

\section{Seed germination, root growth, and superoxide assay}

Using the Agrobacterium tumefaciens GV3101 harboring pCAMBIA 1300-RcDREB2B, wild-type (WT) Arabidopsis (Columbia) was transformed via the floral dipping method [74]. Transgenic Arabidopsis was selected on Murashige and Skoog (MS) medium containing $50 \mathrm{mg} / \mathrm{L}$ hygromycin and confirmed by using PCR analysis. The T3 homozygous positive lines were employed for all further experiments. Vector plants (VC) or transgenic Arabidopsis seeds were sown on MS and plants were grown at $23 \pm 1{ }^{\circ} \mathrm{C}$ under a 16 -h light/8-h dark cycle.

For germination assays, Arabidopsis seeds were surface-sterilized with $75 \%$ alcohol for $5 \mathrm{~min}$ and $2 \%$ $\mathrm{NaClO}$ for $10 \mathrm{~min}$, respectively. To determine the effects of $\mathrm{NaCl}, \mathrm{ABA}$, or PEG on seed germination, seeds from $\mathrm{VC}$ and $\mathrm{T} 3$ transgenic plants (OE\#4, $\mathrm{OE \# 5}$, and $\mathrm{OE} \# 16$ ) were transferred to MS agar plates or MS agar plates saturated with various concentrations of $\mathrm{NaCl}(0,50$, $100,150$ and $200 \mathrm{mM}), \mathrm{ABA}(0,1.2$, and $1.6 \mu \mathrm{M})$ or PEG $4000(0,4,8,12$ and $16 \%)$, respectively. Then, seeds were incubated at $4{ }^{\circ} \mathrm{C}$ for $72 \mathrm{~h}$ and were transferred to a growth chamber $(16 \mathrm{~h}$ light $/ 8 \mathrm{~h}$ dark photoperiod, approximately $100 \mu \mathrm{mol} \mathrm{m}^{-2} \mathrm{~s}^{-1}$ photosynthetic photon flux) at $23 \pm 1{ }^{\circ} \mathrm{C}$ for germination. After $9 \mathrm{~d}$, the rates of seed germination were recorded. The emerging of radical and greening of cotyledon was considered seed germination.

To determine the growth of root, $6 \mathrm{~d}$ old seedlings were carefully transferred to MS media containing different concentrations of $\mathrm{NaCl}(0,100,150$, or $200 \mathrm{mM})$ or ABA $(0$ or $100 \mu \mathrm{M})$ and incubated for $10 \mathrm{~d}$. The position of the root tips was marked at the time of transfer, and the root growth was measured from this mark after $7 \mathrm{~d}$. The root elongation and lateral root number were analyzed by Image J (http://rsb.info.nih.gov/ij). Three independent biological replicates were conducted for salt or ABA experiments.

Nitro blue tetrazolium (NBT) and hydrogen peroxide by 3,3-diaminobenzidine (DAB) staining were carried out for situ detection of superoxide as described previously [75]. Following $10 \mathrm{~d}$ of growth, $\mathrm{H}_{2} \mathrm{O}_{2}$, and $\mathrm{O}_{2}{ }^{-}$ were found in $R c D R E B 2 B$ transgenic and VC seedlings when immersed for $1 \mathrm{~h}$ in either water (CK) or $200 \mathrm{mM}$ $\mathrm{NaCl}$. Concentrated and $90 \%$ ethanol was then used for treating the seedlings for destaining before 
photographing with a Stemi DV4 light microscope (Carl Zeiss, Gottingen, Germany). Ten technical and three biological replicates were used for each tested condition. Mean significant differences were compared by ANOVA analysis at $P \leq 0.05$ utilizing SPSS 24.0 software (IBM, Armonk, NY, USA).

\section{Supplementary Information}

The online version contains supplementary material available at https:/doi. org/10.1186/s12864-021-07396-6.

Additional file 1: Table S1. Information of gene ID, physical position, and properties of RCAP2/ERF proteins.

Additional file 2: Table S2. Primer sequences used in this study.

Additional file 3: Figure S1. Multiple sequence alignment between RCDREB2B and other plant DREB proteins. The conserved DREB AP2/ERF domain is seen as the underlined segment. Stars denote the amino acid residues in the AP2/ERF domain that have been reported to be conserved. NLS and AP2-domain are marked with red and blue solid boxes, respectively. The underlined asterisks of red, blue, and black indicate YRG, WLG, and RAYD motif, respectively.

Additional file 4: Figure S2. Morphological phenotypes of VC and RCDREB2B transgenic Arabidopsis. (A) Flowers morphological of VC and $R C D R E B 2 B$ transgenic lines. Bar $=1 \mathrm{~cm}$. (B) The flower diameter, petal length, petal width, and single petal area of VC and RCDREB2B transgenic lines. 3-week-old plants were photographed and the total areas, petal length, and width were determined by using Image J software. Error bars indicate SE $(n=3)$

Additional file 5: Figure S3. Quantities analysis of $\mathrm{H}_{2} \mathrm{O}_{2}(\mathrm{~A})$ and $\mathrm{O}_{2}^{-}$(B) in VC and RCDREB2B transgenic Arabidopsis.

Additional file 6: Figure S4. Germination analysis of $V C$ and $R C D R E B 2 B$ overexpressing lines in response to PEG. (A) Germination of VC and three RCDREB2B transgenic lines on MS plus different concentrations of PEG $4000(0,4,8,12$ and 16\%) after 9 days. (B) Statistical analyses of germination rates indicated in (A).

Additional file 7: Figure S5. The whole gels for each of the cropped gel images shown in Fig. 6a of the main text.

\section{Abbreviations}

TF: Transcription factor; AP2/ERF: APETALA2/ethylene-responsive factor; NLS: Nuclear localization signal; RT-qPCR: Real-time quantitative PCR; ORF: Open reading frame; MS: Murashige and Skoog; NBT: Nitro blue tetrazolium; DAB: 3,3-diaminobenzidine

\section{Acknowledgments}

Not applicable.

\section{Authors' contributions}

LW and ZC conceived and practically designed the experiments; LW, GZ, and WK carried out the experiments, conducted the data analysis. GZ wrote the manuscript; JX revised the manuscript and contributed the plant material. All authors read and approved the final manuscript.

\section{Funding}

This work was supported by grants from the National Key Research and Development Program of China (2018YFD1000400) and Innovative Program for Graduate Student of Qingdao Agricultural University (QYC201922). The funding bodies had no role in the design of the study and collection, analysis, and interpretation of data and in writing the manuscript.

\section{Availability of data and materials}

The RCDREB2B sequence data was deposited in the NCBI GenBank under accession number MH152409.

Ethics approval and consent to participate Not application.
Consent for publication

Not applicable.

\section{Competing interests}

The authors declare that they have no competing interests.

Received: 7 September 2020 Accepted: 19 January 2021

Published online: 28 January 2021

\section{References}

1. Dimova DK, Stevaux O, Frolov MV, Dyson NJ. Cell cycle-dependent and cell cycle-independent control of transcription by the Drosophila E2F/RB pathway. Genes Dev. 2003;17(18):2308-20.

2. Sharma MK, Kumar R, Solanke AU, Sharma R, Tyagi AK, Sharm AK Identification, phylogeny, and transcript profiling of ERF family genes during development and abiotic stress treatments in tomato. Mol Gen Genomics. 2010;284:455-75.

3. Jofuku KD, Omidyar PK, Gee Z, Okamuro JK. Control of seed mass and seed yield by the floral homeotic gene APETALA2. Proc Natl Acad Sci U S A. 2005; 102(8):3117-22

4. Sakuma Y, Liu Q, Dubouzet JG, Abe H, Shinozaki K, Yamaguchi-Shinozaki K. DNA-binding specificity of the ERF/AP2 domain of Arabidopsis DREBs, transcription factors involved in dehydration- and cold-inducible gene expression. Biochem Biophys Res Commun. 2002;290(3):998-1009.

5. Kunst L, Klenz JE, Martinez-Zapater J, Haughn GW. AP2 gene determines the identity of perianth organs in flowers of Arabidopsis thaliana. Plant Cell. 1989:1(12):1195-208

6. Du C, Hu K, Xian S, Liu C, Fan J, Tu J, Fu T. Dynamic transcriptome analysis reveals AP2/ERF transcription factors responsible for cold stress in rapeseed (Brassica napus L.). Mol Gen Genomics. 2016;291(3):1053-67.

7. Mizoi J, Shinozaki K, Yamaguchi-Shinozaki K. AP2/ERF family transcription factors in plant abiotic stress responses. Biochim Biophys Acta. 2012;1819(2): 86-96.

8. Ohme-Takagi $\mathrm{M}$, Shinshi $\mathrm{H}$. Ethylene-inducible DNA binding proteins that interact with an ethylene-responsive element. Plant Cell. 1995;7(2):173-82.

9. Agarwal P, Agarwal PK, Joshi AJ, Sopory SK, Reddy MK. Overexpression of PgDREB2A transcription factor enhances abiotic stress tolerance and activates downstream stress-responsive genes. Mol Biol Rep. 2010;37(2): 1125-35.

10. Wang C, Wang H, Zhang J, Chen S. A seed-specific AP2-domain transcription factor from soybean plays a certain role in regulation of seed germination. Sci China C Life Sci. 2008:51(4):336-45.

11. Raymond O, Gouzy J, Just J, Badouin H, Verdenaud M, Lemainque A, Vergne P, Moja S, Choisne N, Pont C, Carrère S, et al. The Rosa genome provides new insights into the domestication of modern roses. Nat Genet. 2018;50(6): 772-7.

12. Li M, Liu J, Hao J, Feng K, Duan A, Yang Q, Xu Z, Xiong A. Genomic identification of AP2/ERF transcription factors and functional characterization of two cold resistance-related AP2/ERF genes in celery (Apium graveolens L.). Planta. 2019;250:1265-80.

13. Li X, Tao S, Wei S, Ming M, Huang X, Zhang S, Wu J. The mining and evolutionary investigation of AP2/ERF genes in pear (Pyrus). BMC Plant Biol. 2018;18:46.

14. Li H, Wang Y, Wu M, Li L, Li C, Han Z, Yuan J, Chen C, Song W, Wang C. Genome-wide identification of AP2/ERF transcription factors in cauliflower and expression profiling of the ERF family under salt and drought stresses. Front Plant Sci. 2017:8:946.

15. Jin J, Wang M, Zhang H, Khan A, Wei A, Luo D, Gong Z. Genome-wide identification of the AP2/ERF transcription factor family in pepper (Capsicum annuum L.). Genome. 2018:61(9):663-74.

16. Ji A, Luo H, Xu Z, Zhang X, Zhu Y, Liao B, Yao H, Song J, Chen S. Genomewide identification of the AP2/ERF gene family involved in active constituent biosynthesis in Salvia miltiorrhiza. Plant Genome. 2016;9(2):1-11.

17. Lakhwani D, Pandey A, Dhar Y, Bag SK, Trivedi PK, Asif MH. Genome-wide analysis of the AP2/ERF family in Musa species reveals divergence and neofunctionalisation during evolution. Sci Rep. 2016:6(1):18878.

18. Zhang Z, Li X. Genome-wide identification of AP2/ERF superfamily genes and their expression during fruit ripening of chinese jujube. Sci Rep. 2018;8: 15612. 
19. Liu M, Sun W, Ma Z, Zheng T, Huang L, Wu Q, Zhao G, Tang Z, Bu T, Li C, Chen $\mathrm{H}$. Genome-wide investigation of the AP2/ERF gene family in tartary buckwheat (Fagopyum tataricum). BMC Plant Biol. 2019;19(1):84.

20. Zhang C, Shangguan LF, Ma RJ, Sun X, Tao R, Guo L, Korir NK, Yu ML. Genome-wide analysis of the AP2/ERF superfamily in peach (Prunus persica). Genet Mol Res. 2012;11(4):4789-809.

21. Richau KH, Kaschani F, Verdoes M, Pansuriya TC, Niessen S, Stüber K, Colby T, Overkleeft HS, Bogyo M, Hoorn RALV d. Subclassification and biochemical analysis of plant papain-like cysteine proteases displays subfamily-specific characteristics. Plant Physiol. 2012;158(4):1583-99.

22. Cannon SB, Mitra A, Baumgarten A, Young ND, May G. The roles of segmental and tandem gene duplication in the evolution of large gene families in Arabidopsis thaliana. BMC Plant Biol. 2004;4:10.

23. Wang Y, Tang H, DeBarry JD, Tan X, Li J, Wang X, Lee TH, Jin H, Marler B, Kissinger JC, Paterson AH, Guo H. MCScanX: a toolkit for detection and evolutionary analysis of gene synteny and collinearity. Nucleic Acids Res. 2012;40(7):e49.

24. Jiang X, Zhang C, Lv P, Jiang G, Liu X, Dai F, Gao J. RhNAC3, a stressassociated NAC transcription factor, has a role in dehydration tolerance through regulating osmotic stress-related genes in rose petals. Plant Biotechnol J. 2014;12(1):38-48.

25. Reis RR, da Cunha BADB, Martins PK, Martins MTB, Alekcevetch JC, Chalfun A, Andrade AC, Ribeiro AP, Qin F, Mizoi J, Yamaguchi-Shinozaki K, Nakashima K, Carvalho JDC, de Sousa CAF, Nepomuceno AL, Kobayashi AK, Molinari HBC. Induced over-expression of AtDREB2A CA improves drought tolerance in sugarcane. Plant Sci. 2014;221-222:59-68.

26. Bernadette L, Rainer PB, Gaelle R, Janna B, Elmon S, Elke L, Imre ES. Expression of AtWRKY33 encoding a pathogen-or PAMP-responsive WRKY transcription factor is regulated by a composite DNA motif containing W box elements. Mol Plant Microbe Interact. 2007:20(4):420-9.

27. Son GH, Wan JR, Kim HJ, Nguyen XC, Chung WS, Hong JC, Stacey G. Ethylene-responsive element-binding factor 5, ERF5, is involved in chitininduced innate immunity response. Mol Plant Microbe Interac. 2012;25(1): 48-60.

28. Wang H. Promoters from kin 1 and cor6.6, two homologous Arabidopsis thaliana genes: transcriptional regulation and gene expression induced by low temperature, ABA, osmoticum and dehydration. Plant Mol Biol. 1995; 28(4):605-17.

29. Dong HP, Yu H, Bao Z, Guo X, Peng J, Yao Z, Chen G, Qu S, Dong H. The ABI2-dependent abscisic acid signaling controls HrpN-induced drought tolerance in Arabidopsis. Planta. 2005;221(3):313-27.

30. Villaecija-Aguilar JA, Hamon-Josse M, Carbonnel S, Kretschmar A, Schmid C, Dawid C, Bennett T, Gutjahr C. SMAX1/SMXL2 regulate root and root hair development downstream of KAI2-mediated signaling in Arabidopsis. PLoS Genet. 2019;15(8):e1008327.

31. Yang CY. Hydrogen peroxide controls transcriptional responses of ERF73/ HRE1 and ADH1 via modulation of ethylene signaling during hypoxic stress. Planta. 2014;239(4):877-85.

32. Feinbaum RL, Ausubel FM. Transcriptional regulation of the Arabidopsis thaliana chalcone synthase gene. Mol Cell Biol. 1988;8(5):1985-92.

33. Kim HJ, Cho HS, Pak JH, Kwon T, Lee JH, Kim DH, Lee DH, Kim CG, Chung YS. Confirmation of drought tolerance of ectopically expressed AtABF3 gene in Soybean. Mol Cells. 2018;41(7):413-22.

34. Garcia MNM, Cortelezzi II, Fumagalli M, Capiati DA. Expression of the Arabidopsis $A B F 4$ gene in potato increases tuber yield, improves tuber quality and enhances salt and drought tolerance. Plant Mol Biol. 2018;98:137-52.

35. Li Q, Shi XZ, Ye SJ, Wang S, Chan R, Harkness T, Wang H. A short motif in Arabidopsis CDK inhibitor ICK1 decreases the protein level, probably through a ubiquitin-independent mechanism. Plant J. 2016;87(6):617-28.

36. Hao GP, Zhang XH, Wang YQ, Wu ZY, Huang CL. Nucleotide variation in the NCED3 region of Arabidopsis thaliana and its association study with abscisic acid content under drought stress. J Integr Plant Biol. 2010;51(2):175-83.

37. Singh $\mathrm{KB}$, Foley $\mathrm{RC}$, Oñate-Sánchez L. Transcription factors in plant defense and stress responses. Curr Opin Plant Biol. 2002:5(5):430-6.

38. Lata C, Mishra AK, Muthamilarasan M, Bonthala VS, Khan Y, Prasad M. Genome-wide investigation and expression profiling of AP2/ERF transcription factor superfamily in Foxtail Millet (Setaria italica L.). PLoS One. 2014;9(11):e113092.

39. Zhuang J, Peng R, Cheng Z, Zhang J, Cai B, Zhang Z, Gao F, Zhu B, Fu X, Jin $X$, Chen J, Qiao Y, Xiong A, Yao Q. Genome-wide analysis of the putative AP2/ERF family genes in Vitis vinifera. Sci Hortic. 2009;123(1):73-81.
40. Sara EG, Jaina M, Alex B, Sean RE, Aurélien L, Simon CP, Matloob Q, Lorna JR, Gustavo AS, Alfredo S, Erik LLS, Layla H, Lisanna P, Damiano P, Silvio CET, Robert DF. The Pfam protein families database in 2019. Nucleic Acids Res. 2018:47(D1):D427-32.

41. Xu W, Li F, Ling L, Liu A. Genome-wide survey and expression profiles of the AP2/ERF family in castor bean (Ricinus communis L.). BMC Genom. 2013; 14(1):785.

42. Rao G, Sui J, Zeng Y, He C, Zhang J. Genome-wide analysis of the AP2/ERF gene family in Salix arbutifolia. FEBS Open Biochem. 2015;5(1):132-7.

43. Guo B, Wei Y, Xu R, Lin S, Luan H, Lv C, Zhang X, Song X, Xu R. Genomewide analysis of APETALA2/ethylene-responsive factor (AP2/ERF) gene family in barley (Hordeum vulgare L.). PLoS One. 2016;1 (9):e0161322.

44. Qu L, Zhu Y. Transcription factor families in Arabidopsis: major progress and outstanding issues for future research. Curr Opin Plant Biol. 2013;9(5):544-9.

45. Feng J, Liu D, Pan Y, Gong W, Ma L, Luo J, Deng X, Zhu Y. An annotation update via cdna sequence analysis and comprehensive profiling of developmental, hormonal or environmental responsiveness of the Arabidopsis AP2/EREBP transcription factor gene family. Plant Mol Biol. 2005; 59(6):853-68.

46. Manosalva PM, Davidson RM, Liu B, Zhu X, Hulbert SH, Leung H, Leach JE. A germin-like protein gene family functions as a complex quantitative trait locus conferring broad-spectrum disease resistance in rice. Plant Physiol. 2009;149(1):286-96.

47. Flagel LE, Wendel JF. Gene duplication and evolutionary novelty in plants. New Phytol. 2009;183(3):557-64.

48. Fraser JA, Huang JC, Pukkila-Worley R, Alspaugh JA, Mitchell TG, Heitman J. Chromosomal translocation and segmental duplication in Cryptococcus neoformans. Eukaryot Cell. 2013;4(2):401-6.

49. Zhou D. Regulatory mechanism of plant gene transcription by GT-elements and GT-factors. Trends Plant Sci. 1999:4(6):210-4.

50. Narusaka Y, Nakashima K, Shinwari ZK, Sakuma Y, Furihata T, Abe H, Narusaka M, Shinozaki K, Yamaguchi-Shinozaki K. Interaction between two cis-acting elements, ABRE and DRE, in ABA-dependent expression of Arabidopsis $r d 29 \mathrm{~A}$ gene in response to dehydration and high-salinity stresses. Plant J. 2010;34(2):137-48.

51. He C, Yu Z, Teixeira da Silva JA, Zhang J, Liu X, Wang X, Zhang X, Zeng S, Wu K, Tan J, Ma G, Luo J, Duan J. DoGMP1 from Dendrobium officinale contributes to mannose content of water-soluble polysaccharides and plays a role in salt stress response. Sci Rep. 2017;7:41010.

52. Ambawat S, Sharma P, Yadav NR, Yadav RC. MYB transcription factor genes as regulators for plant responses: an overview. Physiol Mol Biol Plants. 2013; 19(3):307-21.

53. Muhammad AA, Krzysztof W, David PK, Holger B, John J. The beet cyst nematode Heterodera schachtii modulates the expression of WRKY transcription factors in Syncytia to favour its development in Arabidopsis roots. PLoS One. 2014;9(7):e102360.

54. Cui M, Zhang W, Zhang Q, Xu Z, Zhu Z, Duan F, Wu R. Induced overexpression of the transcription factor OSDREBZA improves drought tolerance in rice. Plant Physiol Biochem. 2011:49(12):1384-91.

55. Chen M, Wang Q, Cheng X, Xu Z, Li L, Ye X, Xia L, Ma Y. GmDREB2, a soybean DRE-binding transcription factor, conferred drought and high-salt tolerance in transgenic plants. Biochem Biophys Res Commun. 2007;353(2): 299-305.

56. Liu S, Wang X, Wang H, Xin H, Yang X, Yan J, Li J, Tran L-S P, Shinozaki K, Yamaguchi-Shinozaki K, Qin F. Genome-wide analysis of ZmDREB genes and their association with natural variation in drought tolerance at seedling stage of Zea mays L. PLoS Genet. 2013;9(9):e1003790.

57. Hichri I, Muhovski Y, Clippe A, Žižková E, Dobrev PI, Motyka V, Lutts S. SIDREB2, a tomato dehydration-responsive element-binding 2 transcription factor, mediates salt stress tolerance in tomato and Arabidopsis. Plant Cell Environ. 2016:39(1):62-79.

58. Li X, Zhang D, Li H, Wang Y, Zhang Y, Wood AJ. EsDREB2B, a novel truncated DREB2-type transcription factor in the desert legume Eremosparton songoricum, enhances tolerance to multiple abiotic stresses in yeast and transgenic tobacco. BMC Plant Biol. 2014;14(1):44.

59. Zhang Z, Yang Q, Zhang C, Wei L, Yue R, Li G, Lin X, Wang R. A CKDREB1 gene isolated from Caragana korshinskii Kom. enhances Arabidopsis drought and cold tolerance. Braz J Bot. 2019:42(1):1-9.

60. Yang G, Yu L, Zhang K, Zhao Y, Guo Y, Gao C. A ThDREB gene from Tamarix hispida improved the salt and drought tolerance of transgenic tobacco and T.hispida. Plant Physiol Biochem. 2017;113:187-97. 
61. Tang Y, Liu K, Zhang J, Li X, Xu K, Zhang Y, Qi J, Yu D, Wang J, Li C. $J C D R E B 2$, a physic nut AP2/ERF gene, alters plant growth and salinity stress responses in transgenic rice. Front Plant Sci. 2017;8:306.

62. Qin F, Sakuma Y, Phan Tran LS, Maruyama K, Kidokoro S, Fujita Y, Fujita M, Umezawa T, Sawano Y, Miyazono K-I, Tanokura M, Shinozaki K, YamaguchiShinozaki K. Arabidopsis DREB2A-interacting proteins function as RING E3 ligases and negatively regulate plant drought stress-responsive gene expression. Plant Cell. 2008;20(6):1693-707.

63. Wang L, Waters MT, Smith SM. Karrikin-KAI2 signaling provides Arabidopsis seeds with tolerance to abiotic stress and inhibits germination under conditions unfavorable to seedling establishment. New Phycol. 2018;219(2): 605-18.

64. Gasteiger E, Hoogland C, Gattiker A, Duvaud SE, Wilkins MR, Appel RD, Bairoch A. Protein identification and analysis tools on the ExPASy server. 2-D Proteome Anal Protoc. 2005:112:531-52.

65. Paul H, Keun-Joon P, Takeshi O, Naoya F, Hajime H, Collier CJ, Kenta N WoLF PSORT: protein localization predictor. Nucleic Acids Res. 2007;35: W585-7.

66. Thompson JD, Gibson TJ, Higgins DG. Multiple sequence alignment using ClustalW and ClustalX. Curr Protoc Bioinformatics. 2003;00(1):2.3.1-2.3.22.

67. Bailey TL, Mikael B, Buske FA, Martin F, Grant CE, Luca C, Ren J, Li W, Noble WS. MEME suite: tools for motif discovery and searching. Nucleic Acids Res. 2009;37(2):W202-8

68. Voorrips RE. MapChart: software for the graphical presentation of linkage maps and QTLs. J Hered. 2002;93(1):77-8.

69. Sudhir K, Glen S, Koichiro T. MEGA7: molecular evolutionary genetics analysis version 7.0 for bigger datasets. Mol Biol Evol. 2016;33(7):1870-4.

70. Subramanian B, Gao S, Lercher MJ, Hu S, Chen WH. Evolview v3: a webserver for visualization, annotation, and management of phylogenetic trees. Nucleic Acids Res Issue. 2019;47(W1):W270-5.

71. Lescot M, Dehais P, Thiis G, Marchal K, Moreau Y, Van de Peer Y, Rouzé P, Rombauts S. PlantCARE, a database of plant cis-acting regulatory elements and a portal to tools for in silico analysis of promoter sequences. Nucleic Acids Res. 2002;30(1):325-7.

72. Livak K, Schmittgen TD. Analysis of relative gene expression data using realtime quantitative PCR and the $2^{-\Delta \Delta C T}$ method. Methods. 2001;25:402-8.

73. Yoo S, Cho Y, Sheen J. Arabidopsis mesophyll protoplasts: a versatile cell system for transient gene expression analysis. Nat Protoc. 2007;2:1565-72.

74. Clough SJ, Bent AF. Floral dip: a simplified method for Agrobacteriummediated transformation of Arabidopsis thaliana. Plant J. 1999;16(6):735-43.

75. Shi J, Fu X, Peng T, Huang X, Fan Q, Liu J. Spermine pretreatment confers dehydration tolerance of citrus in vitro plants via modulation of antioxidative capacity and stomatal response. Tree Physiol. 2010;30(7):914-22.

\section{Publisher's Note}

Springer Nature remains neutral with regard to jurisdictional claims in published maps and institutional affiliations.

Ready to submit your research? Choose BMC and benefit from:

- fast, convenient online submission

- thorough peer review by experienced researchers in your field

- rapid publication on acceptance

- support for research data, including large and complex data types

- gold Open Access which fosters wider collaboration and increased citations

- maximum visibility for your research: over $100 \mathrm{M}$ website views per year

At $\mathrm{BMC}$, research is always in progress.

Learn more biomedcentral.com/submissions 